\title{
Fock Space Representations of Affine Lie Algebras and Integral Representations in the Wess-Zumino-Witten Models
}

\author{
Gen Kuroki \\ Mathematical Institute, Tohoku University, Sendai 980, Japan \\ Received January 25, 1991
}

\begin{abstract}
Fock space representations of affine Lie algebras are studied. Explicit forms of correction terms adding to the currents $F_{i}(z)$ are determined. It is proved that the Sugawara energy-momentum tensor on the Fock spaces is quadratic in free bosons. Furthermore, screening operators are constructed. This implies the existence of generalized hypergeometric integrals satisfying the KnizhnikZamolodchikov equation.
\end{abstract}

\section{Table of Contents}

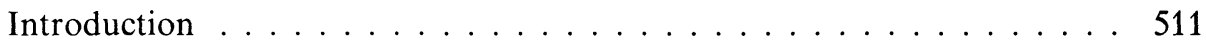

1. Representations of Simple Lie Algebras . . . . . . . . . . . . . . . . 519

2. Bosonic Free Fields and the Wick Theorem . . . . . . . . . . . . 521

3. Lie Algebra Cohomologies . . . . . . . . . . . . . . . . . . 526

4. Fock Space Representations of Affine Lie Algebras . . . . . . . . . . . 530

5. Screening Operators and Integral Representations . . . . . . . . . . . . 534

References ......................... 541

\section{Introduction}

Studies of integral representations in conformal field theories are initiated in $[\mathrm{DF} 1,2]$. Following the earlier paper [FeFu1,2], Dotsenko and Fateev found that conformal blocks in the minimal models introduced in [BPZ] can be represented by generalized hypergeometric integrals. (Throughout the present paper, conformal blocks are those in genus 0 .) The paper [TK1] is closely related to this result. Recently, Felder [Fel] has constructed Fock space resolutions of irreducible representations of the Virasoro algebra and made the physical argument in $[\mathrm{DF} 1,2]$ precise. His work is also based on the very deep results in $[\mathrm{FeFu1,2}]$ 
on representations of the Virasoro algebra. The above studies start from the existence of Fock space representations and screening operators for the Virasoro algebra. In the Wess-Zumino-Witten models, the following problems are fundamental for integral representations:

(a) Construction of Fock space representations of affine Lie algebras.

(b) Realization, by free bosonic fields, of the Sugawara energy-momentum tensor on the Fock spaces.

(c) Construction of screening operators.

(d) Construction of generalized hypergeometric integrals satisfying the KnizhnikZamolodchikov equations.

In [KZ], the Knizhnik-Zamolodchikov (KZ, for short) equations are obtained by rewriting the Sugawara construction of an energy-momentum tensor in the setting of conformal field theory. This is the reason why it is necessary to consider the problem (b). It is widely known that appropriate solutions to the first three problems lead to that of (d) by standard deduction. In the present paper, we solve these problems for the affine Lie algebra attached to an arbitrary simple Lie algebra.

We shall now briefly review some known results about integral representations in the Wess-Zumino-Witten (WZW, for short) models. For the first time, in [CF], Christe and Flüme succeeded in writing down certain integrals satisfying the $\mathrm{sl}_{2}$ $\mathrm{KZ}$ equations for four point functions. The integrals in [CF] are the special cases of the generalized hypergeometric functions studied in the pioneering works [A1,2] and [VGZ]. This part has been recently generalized in [DJMM, Mat and SV]. The case for $\mathrm{Sl}_{2} N$ point functions has been obtained in [DJMM] and the case for $s l_{n} N$ point functions in [Mat]. In [SV], Schechtman and Varchenko have succeeded in constructing generalized hypergeometric integrals satisfying the $\mathrm{KZ}$ equations attached to arbitrary Kac-Moody algebras as well as arbitrary simple Lie algebras. These results are obtained without Fock space representations of affine Lie algebras, which are treated in the following studies. Fock space representations of $\hat{s l}_{2}$ were constructed by Wakimoto [W]. Constructing screening operators for $\hat{s l}_{2}$, Marshakov [Mar] has given another proof of the results in [CF]. Fock space representations of $\hat{s p}_{2} \cong \widehat{s o}_{5}$ as well as of $\hat{s l}_{n}$ are constructed in [GMMOS]. Recently, in the remarkable papers [FeFr1,2], Feigin and Frenkel have proved the existence of Fock space representations of arbitrary affine Lie algebras. In particular, for $\hat{s l}_{n}$, they have explicitly constructed Fock space representations and screening operators. Note that, using this, we can also solve the problem (b) for $\hat{s l}_{n}$. Hence the results in [Mar] and [FeFr1,2] imply those in [DJMM] and [Mat], respectively. Fock space resolutions of irreducible representations of affine Lie algebras are treated in [BF, FeFr1, 2 and BMP]. These are related to integral representations in higher genus Riemann surfaces and quantum group structures in the conformal field theory. However, these parts are not treated in the present paper.

As mentioned above, the problem (a) has been already solved in $[\mathrm{FeFr} 1,2]$. However, in order to solve the other problems (b), (c) and (d), we need more precise analysis of Fock space representations of affine Lie algebras. The most important points are the following: 
(i) Explicit expressions of current operators by free bosons will be very complicated in general. Avoid direct computations using them. Manipulate only general relations obtained by general arguments.

(ii) In all steps, treat not only an affine Lie algebra but also the Virasoro algebra simultaneously.

Of course, (i) is important for finding what is essential. Under the treatment (ii), we can also use the same method in [FeFr1,2]. Thus we can construct Fock space representations of the affine-Virasoro algebras attached to simple Lie algebras. However (ii) is crucial for our argument. Because the Virasoro algebra is very useful not only for solving the problems (b) and (c) but also for determining the explicit expressions of correction terms for current operators.

In the present paper, for simplicity, if we say $A$ is an algebra or a vector space, then $A$ is one over the field $\mathbb{C}$ of complex numbers. However, all the results, in the present paper, except for those about integral representations also hold over an arbitrary field of characteristic zero. We shall often use the notation $\mathbb{N}=\{0,1,2, \ldots\}$ and $\mathbb{Z}=\{0, \pm 1, \pm 2, \ldots\}$. We denote by $U(\mathfrak{a})$ the universal enveloping algebra of a Lie algebra $a$.

0.1. First we shall prepare the notation of a simple Lie algebra and its representations. Let $g$ be a finite dimensional simple Lie algebra, $\left\{H_{i}, E_{i}, F_{i} \mid i=1, \ldots, r\right\}$ its Chevalley generators, and $\left\{\alpha_{1}, \ldots, \alpha_{r}\right\}$ the set of simple roots of $\mathfrak{g}$. Let $\mathfrak{h}, \mathfrak{n}_{+}$and $\mathrm{n}_{-}$denote the subalgebras of $\mathrm{g}$ generated by $\left\{H_{i}\right\},\left\{E_{i}\right\}$ and $\left\{F_{i}\right\}$, respectively. Put $\mathfrak{b}_{ \pm}$equal to the subalgebras $\mathfrak{h} \oplus \mathfrak{n}_{ \pm}$of $\mathfrak{g}$. Let $\lambda$ be a Lie algebra homomorphism from $\mathfrak{b}_{-}$to the 1-dimensional Abelian Lie algebra $\mathbb{C}$. The set of all such $\lambda$ 's can be identified with the dual vector space $\mathfrak{h}^{*}$ of $\mathfrak{h}$. We identify $\mathfrak{h}$ and $\mathfrak{h}^{*}$ by the Killing form $(\cdot \mid \cdot)$ of $\mathfrak{g}$. Denote by $G$ the algebraic group corresponding to $\mathfrak{g}$ and let $\boldsymbol{B}_{ \pm}$ and $N_{ \pm}$be the subgroups of $G$ corresponding to $b_{ \pm}$and $n_{ \pm}$, respectively. Denote by $F$ the flag manifold $B_{-} \backslash G$ and put $o=B_{-} \in F$. Let $\Delta_{+}=\left\{\beta_{1}, \ldots, \beta_{s}\right\}$ be the set of positive roots of $g$ and $\left\{e_{\alpha} \mid \alpha \in \Delta_{+}\right\}$a root basis of $n_{+}$. Then we have the isomorphism $f$ from $\mathbb{C}^{s}$ onto the open cell $o N_{+}$in $F$ defined by $\left(z_{\alpha}\right)_{\alpha \in \Delta_{+}} \mapsto o \exp \left(z_{\beta_{1}} e_{\beta_{1}}\right) \cdots \exp \left(z_{\beta_{s}} e_{\beta_{s}}\right)$. Denote by $x=\left(x_{\alpha}\right)_{\alpha \in \Delta_{+}}$the coordinate system of $o N_{+}$given by the inverse of $f$. Thus the structure ring of $o N_{+}$is identified with the polynomial algebra $\mathbb{C}[x]$. Let $R_{\lambda}$ be the left representation of $\mathfrak{g}$ given by the right infinitesimal action of $\mathfrak{g}$ on $o N_{+}$and the character $\lambda$. We use the notation $M_{\lambda}^{*}$ for the left $\mathfrak{g}$-module $\left(\mathbb{C}[x], R_{\lambda}\right)$. (In the Sect. 1 , we shall denote by $v_{\lambda}$ the element 1 in $\left.M_{\lambda}^{*}=\mathbb{C}[x]\right)$. We remark that Fock space representations of affine Lie algebras will be defined as an affinization of $M_{\lambda}^{*}$. It is easy to show that $M_{\lambda}^{*}$ is isomorphic to the dual of the right Verma module $M_{\lambda}^{\dagger}$ of $\mathfrak{g}$. Therefore, if we put $\lambda=\lambda_{1}+\lambda_{2}$ for $\lambda_{1}, \lambda_{2} \in \mathfrak{h}^{*}$, then we have a canonical $\mathfrak{g}$-homomorphism from $M_{\lambda_{1}}^{*} \otimes M_{\lambda_{2}}^{*}$ to $M_{\lambda}^{*}$. An affinization of this homomorphism is nothing but the bosonic vertex operator. For $X \in \mathfrak{g}$, we can represent $R_{\lambda}(X)$ by a differential operator $R\left(X ; x, \partial_{x}, \lambda\right)$ of first order, where we set $\partial_{x}=\left(\frac{\partial}{\partial x_{\alpha}}\right)_{\alpha \in \Delta_{+}}$. Then $R\left(X ; x, \partial_{x}, \lambda\right)$ is a polynomial in $\left(\lambda\left(H_{i}\right)\right)_{i=1}^{r}$, as well as in $X, x$ and $\partial_{x}$. We define a left action of $N_{+}$on $o N_{+}$by $n \cdot(o a)=$ ona for $a, n \in N_{+}$. This action defines another left representation $S$ of ${n_{+}}_{\text {on }} \mathbb{C}[x]$. Similarly, for $X \in \mathrm{n}_{+}$, we can represent $S(X)$ by a polynomial vector field $S\left(X ; x, \partial_{x}\right)$ in $x$. We shall define screening operators as affinizations of $S\left(E_{i}\right)$ for $i=1, \ldots, r$. 
0.2. Next let us introduce free bosonic fields and Fock spaces. Fix a non-zero complex number $\kappa$. We shall introduce an algebra $\hat{\mathscr{A}}=\hat{\mathscr{A}}_{\kappa}$ as follows. Let $\mathscr{A}$ be the algebra with generators

$$
\left\{x_{\alpha}[m], \delta_{\alpha}[m], p_{i}[m] \mid m \in \mathbb{Z}, \alpha \in \Delta_{+}, i=1, \ldots, r\right\}
$$

and the following commutation relations:

$$
\left[\delta_{\alpha}[m], x_{\beta}[n]\right]=\delta_{\alpha, \beta} \delta_{m+n, 0}, \quad\left[p_{i}[m], p_{r}[n]\right]=\kappa\left(H_{i} \mid H_{j}\right) m \delta_{m+n, 0},
$$

and other commutators are trivial. Define $\hat{\mathscr{A}}$ as a certain $\mathbb{Z}$-graded topological algebra including $\mathscr{A}$ as a dense subalgebra. (For detail, see Sect. 2.) Formally we put

$$
\begin{aligned}
x_{\alpha}(z) & :=\sum_{m \in \mathbb{Z}} z^{-m} x_{\alpha}[m], \quad \delta_{\alpha}(z):=\sum_{m \in \mathbb{Z}} z^{-m-1} \delta_{\alpha}[m], \\
p_{i}(z) & :=\sum_{m \in \mathbb{Z}} z^{-m-1} p_{i}[m],
\end{aligned}
$$

which are called bosonic free fields or free bosons. For $H \in \mathfrak{h}$, writing $H$ in the form $\sum_{i=1}^{r} a_{i} H_{i}$ for some $a_{i} \in \mathbb{C}$, we put $p(H ; z):=\sum_{i=1}^{r} a_{i} p_{i}(z)$ and define $p[H ; m]$ by the expansion $p(H ; z)=\sum_{m \in \mathbb{Z}} z^{-m-1} p[H ; m]$. Let $\lambda$ be in $\mathfrak{h}^{*}$. The Fock space $\mathscr{F}_{\lambda}$ is defined as a left $\mathscr{A}$-module generated by $|\lambda\rangle$ with the following properties:

$$
\begin{aligned}
p_{i}[0]|\lambda\rangle & =\left(\lambda \mid H_{i}\right)|\lambda\rangle, \quad p_{i}[m]|\lambda\rangle=0 \text { for } \quad m>0 \text { and } i=1, \ldots, r, \\
x_{\alpha}[m]|\lambda\rangle & =0, \quad \delta_{\alpha}[n]|\lambda\rangle=0 \quad \text { for } m>0, \quad n \geqq 0 \text { and } \alpha \in \Delta_{+} .
\end{aligned}
$$

These conditions uniquely determine $\mathscr{F}_{\lambda}$ up to isomorphisms. Furthermore $\hat{\mathscr{A}}$ naturally acts on $\mathscr{F}_{\lambda}$.

0.3. Under the above preparation, let us construct Fock space representations of affine Lie algebras. In general, we denote by $L a$ the loop Lie algebra attached to a Lie algebra $\mathfrak{a}$ defined by $L \mathfrak{a}:=\mathfrak{a} \otimes \mathbb{C}\left[t, t^{-1}\right]$. We denote by $\boldsymbol{D}$ the Lie algebra $\mathbb{C}\left[t, t^{-1}\right] \frac{d}{d t}$ of polynomial vector fields on the circle. Then we have the natural semi-direct product $L a \oplus D$ as a Lie algebra. We define the affine-Virasoro algebra $\hat{\mathfrak{g}} \oplus$ Vir attached to $\mathfrak{g}$ as the central extension of $L \mathfrak{g} \oplus \mathfrak{D}$ by $\mathbb{C} K \oplus \mathbb{C} C$ with the following relations:

$$
\begin{gathered}
{[X \otimes f, Y \otimes g]=[X, Y] \otimes f g+(X \mid Y) \operatorname{Res}_{t=0}\left(f^{\prime} g d t\right) K,} \\
{\left[f \frac{d}{d t}, g \frac{d}{d t}\right]=\left(f^{\prime} g-g^{\prime} f\right) \frac{d}{d t}+\frac{1}{12} \operatorname{Res}_{t=0}\left(f^{\prime \prime \prime} g d t\right) C,} \\
{\left[f \frac{d}{d t}, X \otimes g\right]=0 \text { for } X, Y \in g, \text { and } f, g \in \mathbb{C}\left[t, t^{-1}\right],}
\end{gathered}
$$

where the prime' denotes the derivation with respect to $t$. An eigenvalue of $K$ (respectively $C$ ) on a representation space is called a level (respectively a central charge). Now we shall define current operators and an energy-momentum tensor. Roughly speaking, the current operator attached to $X \in \mathfrak{g}$ shall be defined by a 
substitution of $x(z)=\left(x_{\alpha}(z)\right)_{\alpha \in \Delta_{+}}, \delta(z)=\left(\delta_{\alpha}(z)\right)_{\alpha \in \Delta_{+}}$and $p(z)=\left(p_{i}(z)\right)_{i=1}^{r}$ for $x, \partial_{x}$ and $\lambda$ in $R\left(X ; x, \partial_{x}, \lambda\right)$. Denote by $\left\{\Lambda_{i}\right\}_{i=1}^{r}$ the dual basis of $\left\{H_{i}\right\}_{i=1}^{r}$. Put $2 \rho:=\sum_{\alpha \in \Delta_{+}} \alpha$.

For brevity, we often denote by $\partial A(z)$ the derivation of $A(z)$ with respect to $z$. Formally we put

$$
\begin{aligned}
& X(z):=: R(X ; x(z), \delta(z), p(z))^{\bullet} \text { for } X=H_{i}, E_{i} \text { and } i=1, \ldots, r, \\
& F_{i}(z):=: R\left(F_{i} ; x(z), \delta(z), p(z)\right)_{0}^{\bullet}+\gamma_{i} \partial x_{\alpha_{i}}(z) \text { for } i=1, \ldots, r, \\
& T(z):=\sum_{\alpha \in \Delta_{+}}: \delta_{\alpha}(z) \partial x_{\alpha}(z)^{\bullet}+\frac{1}{2 \kappa}\left\{\sum_{i=1}^{r}: p\left(H_{i} ; z\right) p\left(\Lambda_{i} ; z\right)^{\bullet}-\partial p(2 \rho ; z)\right\},
\end{aligned}
$$

where: : denotes a certain normal product (see Sect. 2) and $\left\{\gamma_{i}\right\}_{i=1}^{r}$ is a set of constants which will be fixed in the following theorem. For $X=H_{i}, E_{i}, F_{i}$, the operator $X(z)$ is called the current operator attached to $X$ and $T(z)$ is called the energy-momentum tensor written by free bosons. Then we can define $X[m] \in \hat{A}$ for $X=H_{i}, E_{i}, F_{i}$ and $L_{m} \in \hat{\mathscr{A}}$ by the following formal expansions:

$$
X(z)=\sum_{m \in \mathbb{Z}} z^{-m-1} X[m] \text { and } T(z)=\sum_{m \in \mathbb{Z}} z^{-m-2} L_{m}
$$

Theorem A (Theorem 4.1, Proposition 4.2). There is a unique set $\left\{\gamma_{i}\right\}_{i=1}^{r}$ of constants such that the Lie algebra homomorphism from $\hat{\mathfrak{g}} \oplus \mathrm{Vir}$ to $\hat{\mathscr{A}}$ can be defined by the following:

$$
\begin{gathered}
X \otimes t^{m} \mapsto X[m], \quad t^{m+1} \frac{d}{d t} \mapsto-L_{m}, \\
K \mapsto k=\kappa-g^{*}, \quad C \mapsto c=\frac{k \operatorname{dim} \mathfrak{g}}{k+g^{*}},
\end{gathered}
$$

where $X=H_{i}, E_{i}, F_{i}, m \in \mathbb{Z}$, and $g^{*}$ denotes the dual Coxeter number of $\hat{\mathfrak{g}}$. Moreover the vector $|\lambda\rangle \in \mathscr{F}_{\lambda}$ satisfies the highest weight condition for $\hat{\mathrm{g}} \oplus \mathrm{Vir}$ :

$$
\begin{array}{ll}
H_{i}[0]|\lambda\rangle=\left(\lambda \mid H_{i}\right)|\lambda\rangle, & L_{0}|\lambda\rangle=\Delta_{\lambda}|\lambda\rangle, \quad E_{i}[0]|\lambda\rangle=0, \\
X[m]|\lambda\rangle=L_{m}|\lambda\rangle=0 \quad \text { for } \quad X=H_{i}, E_{i}, F_{i} \quad \text { and } \quad m>0,
\end{array}
$$

where $\Delta_{\lambda}:=(2 \kappa)^{-1}(\lambda \mid \lambda+2 \rho)$.

Denote by $\pi$ the Lie algebra homomorphism given by this theorem. Then we have a family $\left\{\left(\mathscr{F}_{\lambda}, \pi\right)\right\}_{\lambda \in h^{*}}$ of left $\hat{\mathfrak{g}} \oplus$ Vir-modules, which are called the Fock space representations of the affine-Virasoro algebra. (Explicit expressions of the constants $\left\{\gamma_{i}\right\}_{i=1}^{r}$ will be given in Remark 4.3.) As mentioned earlier, the existence of Fock space representations of $\hat{\mathfrak{g}}$ has been already obtained in [FeFr1,2]. However, in $[\mathrm{FeFr} 1,2]$, the explicit expressions of the current operators are described only for $\hat{\mathrm{g}}=\hat{s l}_{n}$. In order to determine the correction terms for $F_{i}(z)$ by $\gamma_{i} \partial x_{\alpha_{i}}(z)$, we shall use the Virasoro operators $\left\{L_{m}\right\}_{m \in \mathbb{Z}}$ (see the proof of Proposition 4.2). Let $\hat{\mathcal{O}}$ be the closed subalgebra of $\hat{\mathscr{A}}$ topologically generated by $\left\{x_{\alpha}[m] \mid \alpha \in \Delta_{+}, m \in \mathbb{Z}\right\}$. For the proof of Theorem $\mathrm{A}$, we shall need certain results about the Lie algebra cohomology of $L \mathfrak{g} \oplus \mathfrak{D}$ with coefficients in $\hat{\mathcal{O}}$ in order to follow the method in $[\mathrm{FeFr} 1,2]$. However, in the present paper, we shall not deal with the result Lie algebra cohomology itself. Instead we shall introduce a certain subcomplex of the 
standard complex so that the homotopy operator $\eta$ in the proof of Lemma 3.2 will be well-defined.

0.4 . A solution to the problem (b) is stated as follows. For any $X \in \mathfrak{g}$, put $X[\mathrm{~m}]:=$ $\pi\left(X \otimes t^{m}\right)$ and $X(z):=\sum_{m \in \mathbb{Z}} z^{-m-1} X[m]$. Let $\left\{J^{p}\right\}_{p=1}^{\operatorname{dim} g}$ be an orthonormal basis of $\mathfrak{g}$ with respect to the Killing form. The Sugawara energy-momentum tensor $T^{\mathrm{SUG}}(z)$ is defined by

$$
T^{\mathrm{SUG}}(z):=\frac{1}{2 \kappa} \sum_{p=1}^{\operatorname{dim} \mathrm{g}}{ }_{\circ}^{\circ} J^{p}(z) J^{p}(z)_{\circ}^{\circ},
$$

where $\stackrel{\circ}{\circ}$ denotes a normal product for currents (see Subsect. 4.4). We write the expansion of this in the form $T^{\text {SUG }}(z)=\sum_{m \in \mathbb{Z}} z^{-m-2} L_{m}^{\text {SUG }}$. Then $L_{m}^{\text {SUG }}$ is well-defined as an operator acting on the Fock spaces.

Theorem B (Theorem 4.5). The energy-momentum tensor $T(z)$ written by free bosons is equal to the Sugawara one on the Fock spaces:

$$
L_{m}=L_{m}^{\text {SUG }} \text { on } \mathscr{F}_{\lambda} \text { for } \lambda \in \mathfrak{h}^{*} \text { and } m \in \mathbb{Z} .
$$

This is deduced from Theorem $\mathrm{A}$ and the fact that, for generic $\lambda \in \mathfrak{h}^{*}$, the Verma module of $\hat{\mathrm{g}}$ with highest weight $\lambda$ is irreducible and isomorphic to $\mathscr{F}_{\lambda}$.

0.5. We can construct screening operators as follows. Let $\lambda$ and $\mu$ be in $\mathfrak{h}^{*}=\mathfrak{h}$. There is a unique linear isomorphism $e^{q[\lambda]}$ from $\mathscr{F}_{\mu}$ onto $\mathscr{F}_{\lambda+\mu}$ with properties

$$
\begin{aligned}
& e^{q[\lambda]}|\lambda\rangle=|\lambda+\mu\rangle, \quad\left[p_{i}[H ; m], e^{q[\lambda]}\right]=\delta_{m, 0}(\lambda \mid H) e^{q[\lambda]}, \\
& {\left[x_{\alpha}[m], e^{q[\lambda]}\right]=\left[\delta_{\alpha}[m], e^{q[\lambda]}\right]=0,}
\end{aligned}
$$

for $H \in \mathfrak{h}, \alpha \in \Delta_{+}$and $m \in \mathbb{Z}$. For brevity, put $\tilde{p}[\lambda ; m]:=\kappa^{-1} p[\lambda ; m]$ for $m \in \mathbb{Z}$. The bosonic vertex operator $V(\lambda ; z)$ is defined by

$$
V(\lambda ; z):=\exp \left\{\sum_{m<0} \frac{z^{-m}}{-m} \tilde{p}[\lambda ; m]\right\} e^{q[\lambda]} z^{\tilde{p}[\lambda ; 0]} \exp \left\{\sum_{m>0} \frac{z^{-m}}{-m} \tilde{p}[\lambda ; m]\right\} .
$$

For $i=1, \ldots, r$, put

$$
S_{i}(z):=: S\left(E_{i} ; x(z), \delta(z)\right)^{*} \quad \text { and } \quad S_{i}(z):=S_{i}(z) V\left(-\alpha_{i} ; z\right) .
$$

If $s_{i}(z)$ is formally expanded in the form $e^{q[\lambda]} \sum_{m \in \mathbb{Z}} s_{i}[m] z^{-m+\tilde{p}[\lambda, 0]}$, then each $s_{i}[m]$ is well-defined as an element of $\hat{\mathscr{A}}$.

Theorem $\mathbf{C}\left(\right.$ Theorem 5.1). For $i=1, \ldots, r$, the operator $s_{i}(z)$ satisfies the following:

$$
\begin{aligned}
{\left[L_{m}, s_{i}(z)\right] } & =\frac{\partial}{\partial z}\left\{z^{m+1} s_{i}(z)\right\} \\
{\left[X[m], s_{i}(z)\right] } & =0 \quad \text { for } \quad X \in \mathfrak{b}_{+}, \\
{\left[F_{j}[m], s_{i}(z)\right] } & =-\kappa \delta_{i, j} \frac{\partial}{\partial z}\left\{z^{m} V\left(-\alpha_{i} ; z\right)\right\} \quad \text { for } \quad j=1, \ldots, r,
\end{aligned}
$$

where $m \in \mathbb{Z}$. 
We call $\left\{s_{i}(z)\right\}_{i=1}^{r}$ the set of screening operators. The first two properties immediately follow from Theorem A. The last property can be deduced from Theorem A and the first two properties.

0.6. Now we have the solutions to the problems (a), (b) and (c). Hence we can obtain certain integrals satisfying the $\mathrm{KZ}$ equations. First let us define the $\mathrm{KZ}$ equations. Recall that $M_{\lambda}^{*}$ denotes the dual of the right $\operatorname{Verma}_{N}$ module $M_{\lambda}^{\dagger}$. Let $\vec{\lambda}=\left(\lambda_{1}, \ldots, \lambda_{N}\right)$ be in $\left(\mathfrak{h}^{*}\right)^{N}$. Put $M_{\bar{\lambda}}^{\dagger}:=\bigotimes_{a=1}^{N} M_{\lambda_{a}}^{\dagger}$ and $M_{\lambda}^{\dagger}:=\bigotimes_{a=1}^{N} M_{\lambda_{a}}^{*}$. Denote by $\langle\mid\rangle$ the natural pairing of $M_{\vec{\lambda}}^{\dagger}$ and $M_{\vec{\lambda}}^{*}$. For $X \in \mathfrak{g}$ and $a=1, \ldots, N$, put

$$
\Delta_{a}(X):=1 \otimes \cdots \otimes 1 \otimes X \otimes 1 \otimes \cdots \otimes 1 \in U(\mathfrak{g})^{\otimes N},
$$

where $X$ is placed at the $a^{\text {th }}$ component. Put $\Delta(X):=\sum_{a=1}^{N} \Delta_{a}(X)$ for $X \in \mathfrak{g}$. Let $\lambda_{\infty}$ be in $\mathfrak{h}^{*}$. We define the weight subspaces of $M_{\bar{\lambda}}^{\dagger}$ and $M_{\lambda}^{*}$ with weight $\lambda_{\infty}$ by

$$
\begin{gathered}
M_{\vec{\lambda}_{, \lambda_{\infty}}}^{\dagger}:=\left\{v^{\dagger} \in M_{\lambda, \lambda_{\infty}}^{\dagger} \mid v^{\dagger} \Delta(H)=v^{\dagger}\left(\lambda_{\infty} \mid H\right) \text { for } H \in \mathfrak{h}\right\}, \\
M_{\lambda_{\lambda, \lambda_{\infty}}^{*}}^{*}:=\left\{v \in M_{\vec{\lambda}, \lambda_{\infty}}^{*} \mid \Delta(H) v=\left(\lambda_{\infty} \mid H\right) v \text { for } H \in \mathfrak{h}\right\} .
\end{gathered}
$$

Then $M_{\lambda, \lambda_{\infty}}^{*}$ is finite dimensional and identified with the dual vector space of $M_{\vec{\lambda}, \lambda_{\infty}}^{\dagger}$. Note that $M_{\bar{\lambda}, \lambda_{\infty}}^{\dagger}$ does not vanish if and only if there exists an $m=\left(m_{i}\right)_{i=1}^{r} \in \mathbb{N}^{r}$ such that

$$
\lambda_{\infty}=\sum_{a=1}^{N} \lambda_{a}-\sum_{i=1}^{r} m_{i} \alpha_{i}
$$

Thus we suppose this in the following. We define the space of singular vectors (or highest weight vectors) in $M_{\vec{\lambda}, \lambda_{\infty}}^{\dagger}$ by

$$
S_{\lambda_{\infty}}^{\dagger}\left(M_{\lambda}^{\dagger}\right):=\left\{v^{\dagger} \in M_{\lambda, \lambda_{\infty}}^{\dagger} \mid v^{\dagger} \Delta\left(\mathrm{n}_{-}\right)=0\right\} .
$$

For $a, b=1, \ldots, N$, the operator $\Omega_{a, b}$ is defined by

$$
\Omega_{a, b}:=\frac{1}{\kappa} \sum_{p=1}^{\operatorname{dim} g} \Delta_{a}\left(J^{p}\right) \Delta_{b}\left(J^{p}\right)
$$

Note that each $\Omega_{a, b}$ preserves the subspace $S_{\lambda_{\infty}}^{\dagger}\left(M_{\vec{\lambda}}^{\dagger}\right)$ of $M_{\vec{\lambda}}^{\dagger}$. The KnizhnikZamolodchikov equation of type $\left(\vec{\lambda}, \lambda_{\infty}\right)$ is written in the following form:

$$
\frac{\partial}{\partial z_{a}} F(z)=\sum_{\substack{1 \leqq b \leqq N \\ b \neq a}} \frac{F(z) \Omega_{a, b}}{z_{a}-z_{b}} \text { for } a=1, \ldots, N
$$

where $z$ denotes $\left(z_{1}, \ldots, z_{N}\right)$ and $F$ is a function of $z$ with values in $S_{\lambda_{\infty}}^{\dagger}\left(M_{\lambda}^{\dagger}\right)$. 0.7 . Next we refer to integration of certain multivalued functions. Put $M:=\sum_{i=1}^{r} m_{i}$
and $t:=\left(t_{1}, \ldots, t_{M}\right)$. Define $\tau=(\tau(1), \ldots, \tau(M))$ by

$$
\tau:=(\underbrace{1, \ldots, 1}_{m_{1} \text { times }}, \ldots, \underbrace{r, \ldots, r}_{m_{r} \text { times }}) \text {. }
$$


For brevity, we use the following abbreviations:

$$
\begin{gathered}
w=\left(w_{1}, \ldots, w_{L}\right):=(z, t)=\left(z_{1}, \ldots, z_{N}, t_{1}, \ldots, t_{M}\right), \\
\vec{\mu}=\left(\mu_{1}, \ldots, \mu_{L}\right):=\left(\lambda_{1}, \ldots, \lambda_{N},-\alpha_{\tau(1)}, \ldots,-\alpha_{\tau(M)}\right),
\end{gathered}
$$

where we put $L:=N+M$. In general, for any $n \in \mathbb{N}$, put $U^{n}:=\left\{\left(\zeta_{1}, \ldots, \zeta_{n}\right) \in \mathbb{C}^{n} \mid\right.$ $\zeta_{a} \neq \zeta_{b}$ if $\left.a \neq b\right\}$. We define the projection $p$ from $U^{L}$ onto $U^{N}$ by $w=(z, t) \mapsto z$. The multivalued function $l(w)=l(z, t)$ in $U^{L}$ is defined by

$$
l(w)=l(z, t):=\prod_{1 \leqq a<b \leqq L}\left(w_{a}-w_{b}\right)^{\left(\mu_{a} \mid \mu_{b}\right) / \kappa} .
$$

Let $\mathscr{L}$ be the 1-dimensional local system on $U^{L}$ defined by $l(w)$ and $\mathscr{L}_{z}$ its restriction on the fiber $p^{-1}(z)$ at $z \in U^{N}$. Denote by $\mathcal{O}\left(U^{L}\right)$ the space of rational functions of $w=(z, t)$ regular in $U^{L}$. For short, we put $d t:=d t_{1} \wedge \cdots \wedge d t_{M}$. For $z \in U^{N}$, let $\Gamma(z)$ be an $M$-cycle in $p^{-1}(z)$ with coefficients in the dual local system $\mathscr{L}_{z}^{*}$ of $\mathscr{L}_{z}$. Then, for $z \in U^{N}$ and a rational function $f(t)$ regular in $p^{-1}(z)$, the integral $\int_{\Gamma(z)} l(z, t) f(t) d t$
is defined and satisfies the following:

$$
\int_{\Gamma(z)} \frac{\partial}{\partial t_{a}}(l(z, t) f(t)) d t=0 \quad \text { for } \quad a=1, \ldots, M .
$$

We suppose that, for every rational function $f(z, t)$ regular in $U^{L}$, the integral $F(z)=\int_{\Gamma(z)}(l(z, t) f(z, t) d t$ is a multivalued holomorphic function of $z$ and satisfies the following:

$$
\frac{\partial}{\partial z_{a}} F(z)=\int_{\Gamma(z)} \frac{\partial}{\partial z_{a}}(l(z, t) f(z, t)) d t \text { for } \quad a=1, \ldots, N .
$$

Note that, in general, for the existence of a non-trivial global family $\{\Gamma(z)\}$, we have to admit $\Gamma(z)$ to be multivalued in $z$.

0.8 . Now, under the above notation, a solution to problem (d) can be stated as follows. Recall that, for each $\lambda \in \mathfrak{h}^{*}$, we identify $M_{\lambda}^{*}$ with the polynomial algebra $\mathbb{C}[x]$ as vector spaces. Thus, for $I_{a}=\left(I_{\alpha}(\alpha)\right)_{\alpha \in \Delta_{+}} \in \mathbb{N}^{\lambda_{+}}$, we can regard $x^{I_{a}}$ as a vector in $M_{\lambda_{a}}^{*}$, where we use the notation $x^{I_{a}}=\prod_{\alpha \in \Delta_{+}} x_{\alpha}^{I_{a}(\alpha)}$ of multi-indices. For $I=\left(I_{a}\right)_{a=1}^{N} \in\left(\mathbb{N}^{\Delta_{+}}\right)^{N}$, put $v^{I}:=\bigotimes_{a=1}^{N} x^{I_{a}} \in M_{\dot{\lambda}}^{*}$. Then the weight subspace $M_{\vec{\lambda}_{\lambda, \lambda_{\infty}}^{*}}$ has the
basis given by

$$
B_{\vec{\lambda}, \mu}^{*}:=\left\{v^{I}\left\{\sum_{a=1}^{N} \sum_{\alpha \in \Delta_{+}} I_{a}(\alpha)=\sum_{i=1}^{r} m_{i} \alpha_{i}\right\} .\right.
$$

Define the $M_{\vec{\lambda}, \lambda_{\infty}}^{\dagger}$-valued function $P(z, t)$ by

$$
\left\langle P(z, t) \mid v^{I}\right\rangle:=\left\langle 0\left|\prod_{a=1}^{N} \prod_{\alpha \in \Delta_{+}} x_{\alpha}\left(z_{a}\right)^{I_{a}(\alpha)} \prod_{b=1}^{m} S_{\tau(b)}\left(t_{b}\right)\right| 0\right\rangle \text { for } \quad v^{I} \in B_{\vec{\lambda}_{, \lambda_{\infty}}}^{*},
$$

where we use the notation of correlation functions of free bosons (for details, see Sect. 5). Then $\left\langle P(z, t) \mid v^{I}\right\rangle$ is a rational function regular in $U^{L}$. 
Theorem D (Theorem 5.9). Under the above notation, define the $M_{\vec{\lambda}_{,},_{\infty}}^{\dagger}$-valued function $F(z)$ by

$$
\langle F(z) \mid v\rangle:=\int_{\Gamma(z)} l(z, t)\langle P(z, t) \mid v\rangle d t \quad \text { for } \quad v \in M_{\vec{\lambda}, \lambda_{\infty}}^{*} .
$$

Then $F(z)$ is valued in $S_{\lambda_{\infty}}^{\dagger}\left(M_{\bar{\lambda}}^{\dagger}\right)$ and satisfies the $\mathrm{KZ}$ equation of type $\left(\vec{\lambda}, \lambda_{\infty}\right)$.

Before finishing this section, we should mention some remarks.

1. There are more essential objects than solutions of the $\mathrm{KZ}$ equations. They are conformal blocks for the Fock spaces, the restrictions of which give solutions of the $\mathrm{KZ}$ equations. The above theorem is obtained as a corollary of the existence of integral representations of conformal blocks for the Fock spaces. See Theorem 5.6 and Lemma 5.7.

2. The origin of the multivalued function $l(w)=l(z, t)$ consists in the following formula for the bosonic vertex operators:

$$
\left\langle\mu_{\infty}\left|\prod_{a=1}^{L} V\left(\mu_{a} ; w_{a}\right)\right| 0\right\rangle=\prod_{1 \leqq a \leqq b \leqq L}\left(w_{a}-w_{b}\right)^{\left(\mu_{a} \mid \mu_{b}\right) / \kappa} \quad \text { up to phase factor, }
$$

where $\mu_{\infty}:=\sum_{a=1}^{L} \mu_{a}$.

3. Denote by $L_{\lambda}^{\dagger}$ the simple right $\mathfrak{g}$-module with highest weight $\lambda \in \mathfrak{h}^{*}$. Put $L_{\lambda}^{\dagger}$ := $\bigotimes_{a=1}^{N} L_{\lambda_{a}}^{\dagger}$. Then $L_{\vec{\lambda}}^{\dagger}$ is naturally a quotient $\mathrm{g}^{\otimes N}$-module of $M_{\dot{\lambda}}^{\dagger}$. We can consider the KZ-equation for the space of singular vectors in $L_{\dot{\lambda}}^{\dagger}$. Let $G(z)$ be the projection of $F(z)$ in $L_{\bar{\lambda}}^{\dagger}$, where $F(z)$ is defined by $(0.32)$. Thus we obtain a solution $G(z)$ of the $\mathrm{KZ}$ equation for the simple $\mathrm{g}$-modules.

\section{Representations of Simple Lie Algebras}

1.1. The notation follows 0.1 in Introduction. For example, $\mathfrak{g}, \mathfrak{h}, \Delta_{+}=\left\{\beta_{1}, \ldots, \beta_{s}\right\}$, and etc. denote a simple Lie algebra, its Cartan subalgebra, the set of positive roots of $\mathfrak{g}$, and etc. In addition, we suppose that the Killing form $(\cdot \cdot)$ is normalized by $(\theta \mid \theta)=2$, where $\theta$ denotes the highest root of $\mathfrak{g}$ (see [Kac, Chapter 7]). Denote by $\Delta$ the set of roots of $\mathfrak{g}$ in $\mathfrak{h}^{*}$. For $\alpha \in \Delta$, let $e_{\alpha}$ be a root vector attached to $\alpha$. We assume, for simplicity, that $e_{\alpha_{i}}=E_{i}$ for $i=1, \ldots, r$.

1.2. Let us define $x=\left(x_{\alpha}\right)_{\alpha \in \Delta_{+}}, R_{\lambda}$ and $S$. For $\lambda \in \mathfrak{h}^{*}$, let $K_{\lambda}^{\dagger}$ be the right ideal of $U(\mathfrak{g})$ generated by $\boldsymbol{n}_{-}$and $\{H-\lambda(H) \cdot 1 \mid H \in \mathfrak{h}\}$. Define the right Verma module $M_{\lambda}^{\dagger}$ of $\mathrm{g}$ with highest weight $\lambda$ by $M_{\lambda}^{\dagger}:=U(\mathfrak{g}) / K_{\lambda}^{\dagger}$ and put $v_{\lambda}^{\dagger}:=1 \bmod K_{\lambda}^{\dagger} \in M_{\lambda}^{\dagger}$. By $R_{\lambda}^{\prime}$ we denote the right representation of $\mathfrak{g}$ on $M_{\lambda}^{\dagger}$ :

$$
v R_{\lambda}^{\prime}(X)=v X \text { for } X \in \mathfrak{g} \text { and } v \in M_{\lambda}^{\dagger} .
$$

Since $M_{\lambda}^{\dagger}$ is canonically isomorphic to $U\left(n_{+}\right)$as right $n_{+}$-modules, we can define the right representation $S^{\prime}$ of $\mathrm{n}_{+}$on $M_{\lambda}^{\dagger}$ by

$$
v_{\lambda}^{\dagger} n S^{\prime}(X):=-v_{\lambda}^{\dagger} X n \text { for } X \in \mathrm{n}_{+} \quad \text { and } n \in U\left(\mathrm{n}_{+}\right)
$$


Putting $M_{\lambda, \mu}^{\dagger}:=\left\{v \in M_{\lambda}^{\dagger} \mid v H=(\mu \mid H) v\right.$ for $H \in \mathfrak{h}$. $\}$ for $\mu \in \mathfrak{h}^{*}$, we obtain the weight space decomposition $M_{\lambda}^{\dagger}=\bigoplus_{\mu \in \mathfrak{h}^{*}} M_{\lambda, \mu}^{\dagger}$. Define the dual $M_{\lambda}^{*}$ of $M_{\lambda}^{\dagger}$ by

$$
M_{\lambda}^{*}:=\bigoplus_{\mu \in h^{*}} \operatorname{Hom}_{\mathbb{C}}\left(M_{\lambda, \mu}^{\dagger}, \mathbb{C}\right)
$$

and denote by $\langle 1\rangle$ the natural pairing of $M_{\lambda}^{\dagger}$ and $M_{\lambda}^{*}$. Then we can define the left representation $R_{\lambda}$ of $g$ on $M_{\lambda}^{*}$ by

$$
\left\langle u \mid R_{\lambda}(X) v\right\rangle=\left\langle u R_{\lambda}^{\prime}(X) \mid v\right\rangle \text { for } u \in M_{\lambda}^{\dagger}, v \in M_{\lambda}^{*} \text { and } X \in \mathfrak{g}
$$

and the left representation $S$ of $n_{+}$on $M_{\lambda}^{*}$ by

$$
\langle u \mid S(Y) v\rangle=\left\langle u S^{\prime}(Y) \mid v\right\rangle \text { for } u \in M_{\lambda}^{\dagger}, v \in M_{\lambda}^{*} \text { and } Y \in \mathfrak{n}_{+} .
$$

We have the basis $\left\{v_{\lambda}^{\dagger} E^{I} \mid I \in \mathbb{N}^{s}\right\}$ of $M_{\lambda}^{*}$, where we use the following abbreviation:

$$
E^{I}=e_{\beta_{1}}^{I_{1}} e_{\beta_{2}}^{I_{2}} \cdots e_{\beta_{s}}^{I_{s}} /\left(I_{1} ! I_{2} ! \cdots I_{s} !\right) \quad \text { for } \quad I=\left(I_{j}\right)_{j=1}^{s} \in \mathbb{N}^{s} .
$$

Denote by $\left\{x^{I} v_{\lambda} \mid I \in \mathbb{N}^{s}\right\}$ the dual basis of $\left\{v_{\lambda}^{\dagger} E^{I} \mid I \in \mathbb{N}^{s}\right\}$ :

$$
\left\langle v_{\lambda}^{\dagger} E^{I} \mid x^{J} v_{\lambda}\right\rangle=\delta_{I, J} \text { for } I, J \in \mathbb{N}^{s} .
$$

Then the natural $\mathrm{g}$-homomorphism from $M_{\lambda+\mu}^{\dagger}$ to $M_{\lambda}^{\dagger} \otimes M_{\mu}^{\dagger}$ induces a $\mathfrak{g}$-homomorphism from $M_{\lambda}^{*} \otimes M_{\mu}^{*}$ to $M_{\lambda+\mu}^{*}$. Thus we obtain the natural algebra structure in $M^{*}=\bigoplus_{\lambda \in h^{*}} M_{\lambda}^{*}$, which is characterized by

$$
x^{I} v_{\lambda} \cdot x^{J} v_{\mu}=x^{I+J} v_{\lambda+\mu} \text { for } I, J \in \mathbb{N}^{s} \text { and } \lambda, \mu \in \mathfrak{h}^{*} .
$$

In other words, the algebra $M^{*}$ is identified with the tensor product of the polynomial algebra in $\left(x_{\alpha}\right)_{\alpha \in \Delta_{+}}$and the group algebra attached to $\mathfrak{h}^{*}$. Hence we can write $M_{\lambda}^{*}=\mathbb{C}[x] v_{\lambda}$.

1.3. In this subsection, we shall summarize some results on the forms of the operators $R_{\lambda}(X)$ for $X \in \mathfrak{g}$ and $S(Y)$ for $Y \in \mathfrak{n}_{+}$. Under the above identification, $R_{\lambda}(X)$ and $S(Y)$ can be written in the following forms:

$$
\begin{aligned}
R_{\lambda}(X)=\sum_{\alpha \in \Delta_{+}} R_{\alpha}(X ; x) \frac{\partial}{\partial x_{\alpha}}+\sum_{i=1}^{r} \rho_{i}(X ; x)\left(\lambda \mid H_{i}\right) & \text { for } \quad X \in \mathfrak{g} \text { and } \lambda \in \mathfrak{h}^{*}, \\
S(Y)=\sum_{\alpha \in \Delta_{+}} S_{\alpha}(Y ; x) \frac{\partial}{\partial x_{\alpha}} & \text { for } Y \in \mathfrak{n}_{+}
\end{aligned}
$$

where $R_{\alpha}(X ; x), \rho_{i}(X ; x)$ and $S_{\alpha}(Y ; x)$ and polynomials in $x=\left(x_{\alpha}\right)_{\alpha \in \Delta_{+}}$. Note that $R_{\alpha}(X ; x), \rho_{i}(X ; x)$ and $S_{\alpha}(Y ; x)$ do not depend on $\lambda$. When $R_{\alpha}(X ; x)$ and $\rho_{i}(X ; x)$ are written in the forms

$$
\begin{array}{rll}
R_{\alpha}(X ; x) & =\sum_{I \in \mathbf{N} s} a_{I}(X) x^{I}, \quad \text { where } & a_{I}(X) \in \mathbb{C}, \\
\rho_{i}(X ; x) & =\sum_{I \in \mathbb{N}^{s}} b_{I}(X) x^{I}, \quad \text { where } & b_{I}(X) \in \mathbb{C},
\end{array}
$$

the coefficients $a_{I}(X)$ and $b_{I}(X)$ are computed by

$$
\begin{aligned}
& a_{I}(X)=\left\langle v_{0}^{\dagger} E^{I} \mid R_{0}(X ; x) x_{\alpha} v_{0}\right\rangle, \\
& b_{I}(X)=\left\langle v_{\Lambda_{i}}^{\dagger} E^{I} \mid R_{\Lambda_{i}}(X) v_{\Lambda_{t}}\right\rangle .
\end{aligned}
$$


The coefficients in $S_{\alpha}(X)$ are also determined by the similar formulas. Using them, we can prove the following lemmas.

Lemma 1.1. For $X \in \mathrm{n}_{+}$and $\alpha \in \Delta_{+}$, the following results hold on the form of $R_{\lambda}(X)$ and $S(X)$ :

(1) $\rho_{i}(X ; x)=0$ for $i=1, \ldots, r$.

(2) $R_{\alpha}(X ; x)$ and $S_{\alpha}(X ; x)$ are polynomials in $\left\{x_{\beta} \mid \beta \in \Delta_{+}\right.$and $\left.\alpha>\beta\right\}$, where $\alpha>\beta$ means that $\alpha \neq \beta$ and $\alpha-\beta=\sum_{i=1} m_{i} \alpha_{i}$ for some $m_{1}, \ldots, m_{r} \in \mathbb{N}$.

(3) $R_{\alpha}\left(e_{\alpha} ; x\right)=-S_{\alpha}\left(e_{\alpha} ; x\right)=1$.

(4) $R_{\alpha_{i}}\left(e_{\alpha} ; x\right)=S_{\alpha_{1}}\left(e_{\alpha} ; x\right)=0$ unless $\alpha=\alpha_{i}$.

Lemma 1.2. For $i, j=1, \ldots, r$ and $\alpha \in \Delta_{+}$, we have $R_{\alpha}\left(H_{i} ; x\right)=-\left(\alpha \mid H_{i}\right) x_{\alpha}$ and $\rho_{j}\left(H_{i} ; x\right)=\delta_{i, j}$ :

$$
R_{\lambda}(H)=-\sum_{\alpha \in \Delta_{+}}(\alpha \mid H) x_{\alpha} \frac{\partial}{\partial x_{\alpha}}+(\lambda \mid H) \text { for } \lambda \in \mathfrak{h}^{*} \text { and } H \in \mathfrak{h} .
$$

Lemma 1.3. For $i, j=1, \ldots, r$, we have $\rho_{j}\left(F_{i} ; x\right)=\delta_{i, j}\left(\lambda \mid H_{i}\right) x_{\alpha_{i}}$.

Lemma 1.4. For $\lambda \in \mathfrak{h}^{*}$, we have the following commutation relations:

$$
\begin{aligned}
{\left[R_{\lambda}(X), S(Y)\right] } & =0 \text { for } X, Y \in \mathfrak{n}_{+}, \\
{\left[R_{\lambda}(H), S\left(e_{\alpha}\right)\right] } & =(\alpha \mid H) S\left(e_{\alpha}\right) \quad \text { for } \quad \alpha \in \Delta_{+} \quad \text { and } H \in \mathfrak{h}, \\
{\left[R_{\lambda}\left(F_{i}\right), S\left(E_{j}\right)\right] } & =\delta_{i, j}\left(\lambda \mid H_{i}\right)+\left(\alpha_{j} \mid H_{i}\right) x_{\alpha_{i}} S\left(E_{j}\right) \quad \text { for } \quad i, j=1, \ldots, r .
\end{aligned}
$$

\section{Bosonic Free Fields and the Wick Theorem}

2.1. Let $\mathscr{A}$ be an algebra with generators (0.1) and relations (0.2). We define the subsets $A_{0}$ and $A_{ \pm}$of $\mathscr{A}$ by

$$
\begin{aligned}
& A_{0}:=\left\{p_{i}[0] \mid i=1, \ldots, r\right\}, \\
& A_{+}:=\left\{x_{\alpha}[m], \delta_{\alpha}[n], p_{i}[m] \mid \alpha \in \Delta_{+}, m>0, n \geqq 0, i=1, \ldots, r\right\}, \\
& A_{-}:=\left\{x_{\alpha}[m], \delta_{\alpha}[n], p_{i}[n] \mid \alpha \in \Delta_{+}, m \leqq 0, n<0, i=1, \ldots, r\right\} .
\end{aligned}
$$

Let $\mathscr{A}_{0}$ and $\mathscr{A}_{ \pm}$be the subalgebras of $\mathscr{A}$ generated by $A_{0}$ and $A_{ \pm}$, respectively. The normal product: : is the linear isomorphism from $\mathscr{A}_{-} \otimes \mathscr{A}_{0} \otimes \mathscr{A}_{+}$onto $\mathscr{A}$ defined by

$$
: a_{-} \otimes a_{0} \otimes a_{+}::=a_{-} a_{0} a_{+} \text {for } a_{0} \in \mathscr{A}_{0} \text { and } a_{ \pm} \in \mathscr{A}_{ \pm} \text {. }
$$

From now on, we omit $\otimes$ in the left-hand side of this.

2.2. Now let us define a topological $\mathbb{Z}$-graded algebra $\hat{\mathscr{A}}$ including $\mathscr{A}$ as a dense subalgebra. Let $D$ be the derivation of $\mathscr{A}$ with the following property:

$$
D a[m]=m a[m] \text { for } a[m]=x_{\alpha}[m], \delta_{\alpha}[m], p_{i}[m] .
$$

For $m \in \mathbb{Z}$, putting

$$
\mathscr{A}[m]:=\{a \in \mathscr{A} \mid D a=m a\} \text { and } \mathscr{A}_{ \pm}[m]:=\mathscr{A}_{ \pm} \cap \mathscr{A}[m] \text {, }
$$



we obtain the decompositions $\mathscr{A}=\bigoplus_{m \in \mathbb{Z}} \mathscr{A}[m]$ and $\mathscr{A}_{ \pm}=\underset{m \geqq 0}{\bigoplus_{ \pm}} \mathscr{A}_{ \pm}[ \pm m]$. Further-
more we obtain

$$
\mathscr{A}[m]=\bigoplus_{i \in \mathbb{Z}} \mathscr{A}_{-}[m-i] \mathscr{A}_{0} \mathscr{A}_{+}[i] \text { for } m \in \mathbb{Z}
$$

We introduce the decreasing filtration $\left\{\mathscr{A}^{n}[m]\right\}_{n \in \mathbb{Z}}$ of $\mathscr{A}[m]$ by

$$
\mathscr{A}^{n}[m]:=\bigoplus_{i \geqq n} \mathscr{A}_{-}[m-i] \mathscr{A}_{0} \mathscr{A}_{+}[i] \text { for } m \in \mathbb{Z} \text { and } n \in \mathbb{N}
$$

Let $\hat{A}[m]$ denote the completion of $\mathscr{A}[m]$ with respect to this filtration:

$$
\hat{A}[m]:=\underset{n \rightarrow \infty}{\operatorname{proj} \lim } \mathscr{A}[m] / \mathscr{A}^{n}[m] \text { for } m \in \mathbb{Z}
$$

Define the vector space $\hat{\mathscr{A}}$ by

$$
\hat{\mathscr{A}}:=\bigoplus_{m \in \mathbb{Z}} \hat{\mathscr{A}}[m] .
$$

Since $\mathscr{A}^{n_{1}}\left[m_{1}\right] \mathscr{A}^{n_{2}}\left[m_{2}\right]$ is included in $\mathscr{A}^{n}\left[m_{1}+m_{2}\right]$ with $n=\max \left\{n_{1}+m_{2}, n_{2}\right\}$, the multiplication map from $\mathscr{A}\left[m_{1}\right] \times \mathscr{A}\left[m_{2}\right]$ to $\mathscr{A}\left[m_{1}+m_{2}\right]$ is continuous under the topologies given by the filtrations. Thus we can obtain the topological $\mathbb{Z}$-graded algebra structure of $\mathscr{A}$. Recall that, for $\lambda \in \mathfrak{h}^{*}$, the Fock space $\mathscr{F}_{\lambda}$ has been defined in 0.2 . The natural representation of $\hat{A}_{\text {on }} \mathscr{F}_{\lambda}$ is induced by that of $\mathscr{A}$ on $\mathscr{F}_{\lambda}$.

2.3. We shall recall the Wick theorem for free bosons. We have defined, by $(0.3)$, the following free bosonic fields:

$$
x_{\alpha}(z), \delta_{\alpha}(z), p_{i}(z), \quad \text { where } \alpha \in \Delta_{+} \text {and } i=1, \ldots, r .
$$

Let each of $a(z), b(z), a_{m}(z)$ and $b_{n}(z)$ be one of the operator in (2.9). Put

$$
A(z):=: \prod_{m=1}^{M} a_{m}(z)^{\circ} \text { and } B(w):=: \prod_{n=1}^{N} b_{n}(z)^{\circ} .
$$

When $A(z)$ is expanded in the form $\sum_{m \in \mathbb{Z}} z^{-m-h} A_{m}$, each $A_{m}$ is well-defined as an element of $\hat{\mathscr{A}}$. Set $\left(h, d_{+}, d_{-}\right):=(0,1,0),(1,0,-1)$ or $(1,1,-1)$ according as $a(z)=x_{\alpha}(z), \delta_{\alpha}(z)$ or $p_{i}(z)$ for some $\alpha \in \Delta_{+}$or $i=1, \ldots, r$. Expanding $a(z)$ in the form $\sum_{m \in \mathbb{Z}} z^{-m-h} a[m]$, we define the annihilation part $a(z)_{+}$and the creation part $a(z)_{-}$ of $a(z)$ by

$$
a(z)_{+}:=\sum_{m \geqq d_{+}} z^{-m-h} a[m] \text { and } a(z)_{-}:=\sum_{m \leqq d_{-}} z^{-m-h} a[m] .
$$

The contraction $\langle a(z) b(w)\rangle$ of $a(z)$ and $b(w)$ is defined by

$$
\langle a(z) b(w)\rangle:=\left[a(z)_{+}, b(w)_{-}\right] .
$$

Note that $\langle a(z) b(w)\rangle$ is a formal series with coefficients in $\mathbb{C}$. In fact, we have the following formulas:

$$
\begin{aligned}
& \left\langle\delta_{\alpha}(z) x_{\beta}(w)\right\rangle=\delta_{\alpha, \beta}[z-w]^{-1}, \\
& \left\langle x_{\alpha}(z) \delta_{\beta}(w)\right\rangle=-\delta_{\alpha, \beta}[z-w]^{-1}, \\
& \left\langle p_{i}(z) p_{j}(w)\right\rangle=\kappa\left(H_{i} \mid H_{j}\right)[z-w]^{-2},
\end{aligned}
$$


where we put formally

$$
[z-w]^{-i-1}:=\left(\sum_{m=0}^{\infty} z^{-m-1} w^{m}\right)^{i+1}=\frac{1}{i !}\left(-\frac{\partial}{\partial z}\right)^{i} \sum_{m=0}^{\infty} z^{-m-1} w^{m} \text { for } i \in \mathbb{N} \text {. }
$$

Note that $[z-w]^{-i-1} \neq(-1)^{i+1}[w-z]^{-i-1}$.

Lemma 2.1 (the Wick theorem). Under the above situation, we have

$$
\begin{aligned}
A(z) B(w) & =: \prod_{m=1}^{M} a_{m}(z)^{\cdot}: \prod_{n=1}^{N} b_{n}(w)^{\cdot} \\
& =\sum_{v=0}^{\infty} \sum^{(v)} \prod_{i=1}^{v}\left\langle m_{i} n_{\sigma(i)}\right\rangle: \prod_{\substack{1 \leqq m \leqq M \\
m \notin I}} a_{m}(z) \prod_{\substack{1 \leqq n \leqq N \\
n \notin J}} b_{n}(z)^{\bullet}
\end{aligned}
$$

where we put $\langle m n\rangle:=\left\langle a_{m}(z) b_{n}(w)\right\rangle$ and the sum $\sum^{(v)}$ runs over the following data:

$$
\left\{\begin{array}{l}
I=\left\{m_{1}, \ldots, m_{v}\right\} \quad \text { with } 1 \leqq m_{1}<\cdots<m_{v} \leqq M, \\
J=\left\{n_{1}, \ldots, n_{v}\right\} \quad \text { with } 1 \leqq n_{1}<\cdots<n_{v} \leqq N, \\
\sigma \in \Xi_{v}=\{\text { permutations of } 1, \ldots, v\} .
\end{array}\right.
$$

The proof is straightforward. Roughly speaking, the Wick theorem says that the product of the two normal products of free fields can be calculated by summing all contributions from the possible combinations of contractions. It is found by (2.13) that the expression for $B(w) A(z)$ is obtained by replacing $[z-w]^{-i-1}$ in (2.15) by $(-1)^{i+1}[w-z]^{-i-1}$.

2.4. Let us explain operator product expansions. Let $C(z, w)$ be a formal Laurent series of $(z, w)$ with coefficients in $\hat{\mathscr{A}}$ such that, for $i \in \mathbb{N}$, the expression $\left[\left(\frac{\partial}{\partial z}\right)^{i} C(z, w)\right]_{z=w}$ is well-defined as a formal series of $w$ with coefficients in $\hat{\mathscr{A}}$. For example, it is the case for $C(z, w)=: \prod_{m=1}^{M} a_{m}(z) \prod_{n=1}^{N} b_{n}(w)$. under the notation in 2.3. Formally we put

$$
\operatorname{Res}_{z=w} \frac{C(z, w) d z}{(z-w)^{i+1}}:=\left[\frac{1}{i !}\left(\frac{\partial}{\partial z}\right) C(z, w)\right]_{z=w} \text { for } i \in \mathbb{N} .
$$

Let $A(z), B(w)$ and $C_{i}(z, w)$ be formal Laurent series in $z, w$ and $(z, w)$ with coefficients in $\hat{A}$. Suppose that each $C_{i}(z, w)$ has the same property of $C(z, w)$. Expand $A(z)$ in the form $\sum_{m \in \mathbb{Z}} z^{-m-h} A_{m}$. If we have

$$
\left[A_{m}, B(w)\right]=\sum_{i=0}^{N} \operatorname{Res}_{z=w} \frac{z^{m+h-1} C_{i}(z, w) d z}{(z-w)^{i+1}} \text { for } m \in \mathbb{Z},
$$

then we write

$$
A(z) B(w) \sim \sum_{i=0}^{N} \frac{C_{i}(z, w)}{(z-w)^{i+1}},
$$

and say that the operator product expansion (OPE, for short) of $A(z) B(w)$ is equal to the right-hand side of this. 
Lemma 2.2. Under the notation in 2.3, if $A(z)$ and $B(w)$ is defined by (2.10), then there is an OPE of $A(z) B(w)$.

This is easily deduced from the Wick theorem. In fact, an OPE of $A(z) B(w)$ can be calculated by substituting $[z-w]^{-i-1}$ in $(2.15)$ for $\frac{1}{(z-w)^{i+1}}$.

Example. For $\alpha, \beta \in \Delta_{+}$and $m, n \in \mathbb{N}$, put $A(z):=:_{\alpha}(z)^{m} \delta_{\beta}(z)^{\bullet}$ and $B(w):=x_{\beta}(w)^{n} \delta_{\alpha}(w)^{\bullet}$. Then, using the Wick theorem (Lemma 2.1), we obtain

$$
\begin{aligned}
A(z) B(w)= & : x_{\alpha}(z)^{m} \delta_{\beta}(z) x_{\beta}(w)^{n} \delta_{\alpha}(w)^{\bullet}+n[z-w]^{-1} \cdot x_{\alpha}(z)^{m} x_{\beta}^{n-1}(w) \delta_{\alpha}(w): \\
& -m[z-w]^{-1} \cdot x_{\alpha}(z)^{m-1} \delta_{\beta}(z) x_{\beta}(w)^{n} \cdot-m n[z-w]^{-2} \cdot x_{\alpha}(z)^{m-1} x_{\beta}(w)^{n-1} .
\end{aligned}
$$

Hence the OPE of $A(z)$ and $B(w)$ is written in the following form:

$$
\begin{aligned}
A(z) B(w) \sim & \frac{n \cdot x_{\alpha}(z)^{m} x_{\beta}^{n-1}(w) \delta_{\alpha}(w)^{\bullet}-m \cdot x_{\alpha}(z)^{m-1} \delta_{\beta}(z) x_{\beta}(w)^{n}}{z-w} \\
& -\frac{m n \cdot x_{\alpha}(z)^{m-1} x_{\beta}(w)^{n-1} \cdot}{(z-w)^{2}} \quad \square
\end{aligned}
$$

2.5. Let $\hat{\mathcal{O}}$ be the closed subalgebra of $\hat{\mathscr{A}}$ topologically generated by $\left\{x_{\alpha}[m] \mid \alpha \in \Delta_{+}\right.$, $m \in \mathbb{Z}\}$. The Lie algebra $L \mathfrak{g} \oplus \mathfrak{D}$ has been defined in 0.3 . We shall introduce an action of $L \mathfrak{g} \oplus \boldsymbol{D}$ on $\hat{\mathcal{O}}$ and certain 2-cocycles of $L \mathfrak{g} \oplus \mathfrak{d}$ with coefficients in $\hat{\mathcal{O}}$. Put

$$
\tilde{X}(z):=\sum_{\alpha \in \Delta_{+}}: R_{\alpha}(X ; x(z)) \delta_{\alpha}(z):+\sum_{i=1}^{r} \rho_{i}(X ; x(z)) p_{i}(z) \text { for } X \in \mathfrak{g},
$$

where we use the notation $x(z):=\left(x_{\alpha}(z)\right)_{\alpha \in \Delta_{+}}$. Define the energy-momentum tensor $T(z)$ by $(0.8)$.

Lemma 2.3. We have the following OPE's:

$$
\begin{aligned}
& \tilde{X}(z) \tilde{Y}(w) \sim \frac{[\tilde{X, Y}](w)}{z-w}+\frac{\Omega_{1}(X, Y ; z, w)}{(z-w)^{2}} \quad \text { for } \quad X, Y \in \mathfrak{g}, \\
& T(z) \tilde{X}(w) \sim \frac{\frac{\partial}{\partial w} \tilde{X}(w)}{z-w}+\frac{\tilde{X}(w)}{(z-w)^{2}}+\frac{\Omega_{2}(X ; w)}{(z-w)^{3}} \text { for } X \in \mathfrak{g}, \\
& T(z) T(w) \sim \frac{\frac{\partial}{\partial w} T(w)}{z-w}+\frac{2 T(w)}{(z-w)^{2}}+\frac{c / 2}{(z-w)^{4}},
\end{aligned}
$$

where we put

$$
\begin{aligned}
\Omega_{1}(X, Y ; z, w):= & -\sum_{\alpha, \beta \in \Delta_{+}}: \frac{\partial}{\partial x_{\beta}} R_{\alpha}(X ; x(z)) \frac{\partial}{\partial x_{\alpha}} R_{\beta}(Y ; x(w)) \\
& +\sum_{i, j=1}^{r} \kappa\left(H_{i} \mid H_{j}\right) \rho_{i}(X ; x(z)) \rho_{j}(Y ; x(w)),
\end{aligned}
$$




$$
\begin{aligned}
\Omega_{2}(X ; w) & :=\sum_{\alpha \in \Delta_{+}}: \frac{\partial}{\partial x_{\alpha}} R_{\alpha}(X ; x(w))_{\bullet}^{\cdot}+2 \sum_{j=1}^{r} \rho_{j}(X ; x(w)), \\
c: & =\operatorname{dim} \mathfrak{g}-12(\rho \mid \rho) / \kappa . \quad \square
\end{aligned}
$$

The proof is straightforward by the Wick theorem (Lemma 2.1). We remark that $c=\left(\kappa-g^{*}\right) \operatorname{dim} g / \kappa$ because of the strange formula $g^{*} \operatorname{dim} g=12(\rho \mid \rho)$, where $g^{*}$ is the dual Coexter number of $\hat{\mathrm{g}}$.

The linear map $\tilde{\pi}$ from $L \mathfrak{g} \oplus \mathfrak{d}$ to $\hat{\mathscr{A}}$ is defined by the following expansions:

$$
\begin{aligned}
& \tilde{X}(z)=\sum_{m \in \mathbb{Z}} z^{-m-1} \tilde{\pi}\left(X \otimes t^{m}\right) \quad \text { for } \quad X \in \mathfrak{g}, \\
& T(z)=\sum_{m \in \mathbb{Z}} z^{-m-2} \tilde{\pi}\left(-t^{m+1} \frac{d}{d t}\right) .
\end{aligned}
$$

Owing to the Wick theorem, we can define the representation of $L \mathfrak{g} \oplus \mathfrak{D}$ on $\hat{\mathcal{O}}$ by

$$
a \cdot f:=[\tilde{\pi}(a), f] \text { for } a \in L \mathfrak{g} \oplus \mathcal{D} \text { and } f \in \hat{\mathcal{O}} .
$$

We define the linear map $\omega$ from $\wedge^{2}\left(L \mathfrak{b}_{+} \oplus \mathfrak{d}\right)$ to $\hat{\mathscr{A}}$ by

$$
\omega(a, b):=[\tilde{\pi}(a), \tilde{\pi}(b)]-\tilde{\pi}([a, b]) \text { for } \quad a, b \in L \mathfrak{g} \oplus \boldsymbol{D} .
$$

By definition, $\omega$ satisfies the cocycle condition. Since $\Omega_{1}$ and $\Omega_{2}$ are formal series with coefficients in $\hat{\mathcal{O}}$, the 2 -cocycle $\omega$ is valued in $\hat{\mathcal{O}}$. Thus we obtain the 2 -cocycle $\omega$ of $L \mathfrak{g} \oplus \mathcal{D}$ with coefficients in $\hat{\mathcal{O}}$. In addition, we define the standard 2-cocycle $c_{2}$ of $L \mathfrak{g} \oplus \mathfrak{D}$ with coefficients in $\mathbb{C} \subset \hat{\mathcal{O}}$ by the following:

$$
\begin{aligned}
c_{2}(X \otimes f, Y \otimes g) & =k \operatorname{Res}_{t=0}\left(f^{\prime} g d t\right), \\
c_{2}\left(f \frac{d}{d t}, g \frac{d}{d t}\right) & :=\frac{c}{12} \operatorname{Res}_{t=0}\left(f^{\prime \prime \prime} g d t\right), \\
c_{2}\left(f \frac{d}{d t}, X \otimes g\right) & =0 \text { for } X, Y \in \mathfrak{g} \text { and } f, g \in \mathbb{C}\left[t, t^{-1}\right],
\end{aligned}
$$

where we put $\dot{k}:=\kappa-g^{*}$. The standard 2-cocycle $c_{2}$ is nothing but the 2-cocycle given by the level $k$ affine Lie algebra and the Sugawara construction of the Virasoro operators. In Sect. 4 , we shall prove that $\omega$ and $c_{2}$ give the same cohomology class.

2.6. Let us summarize some results on $\omega$ immediately following from Lemma 2.3 .

Lemma 2.4. The restriction of $\omega$ on $\wedge^{2}\left(L b_{+} \oplus \mathfrak{D}\right)$ coincides with that of $c_{2}$.

Proof. It follows from (2.25) that $\omega=c_{2}$ on $\wedge^{2} \mathfrak{d}$. Applications of Lemmas 1.1 and 1.2 to (2.23) show that $\omega=c_{2}$ on $\wedge^{2} L n_{+}$. It is deduced from Lemma 1.1 and (2.24)

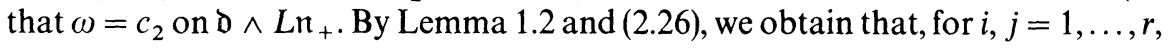

$$
\Omega_{1}\left(H_{i}, H_{j} ; z, w\right)=-\sum_{\alpha, \beta \in \Delta_{+}}\left(\alpha \mid H_{i}\right)\left(\beta \mid H_{j}\right)+\kappa\left(H_{i} \mid H_{j}\right)=\left(\kappa-g^{*}\right)\left(H_{i} \mid H_{j}\right) .
$$

This means that $\omega=c_{2}$ on $\wedge^{2} L \mathfrak{h}$. By Lemma 1.2 and (2.27), we obtain that, for 
$i=1, \ldots, r$

$$
\Omega_{2}\left(H_{i} ; w\right)=-\sum_{\alpha \in \Delta_{+}}\left(\alpha \mid H_{i}\right)+2=-2\left(\rho \mid H_{i}\right)+2=0 .
$$

Thus $\omega=c_{2}$ on $\mathfrak{d} \wedge L \mathfrak{h}$.

Lemma 2.5. For every $X \in \mathfrak{g}$ and $H \in \mathfrak{h}$, the formal series $\Omega_{1}(H, X ; z, w)$ does not contain the formal variable $z$.

This is immediately deduced from Lemmas 1.2 and 2.3 .

\section{Lie Algebra Cohomologies}

3.1. In this section, we shall introduce a certain cohomology $\tilde{H}^{\cdot}(\mathscr{L}, V)$ of a $\mathbb{Z}$-graded Lie algebra $\mathscr{L}$ with coefficients in a $\mathbb{Z}$-graded $\mathscr{L}$-module $V$. We shall define $\tilde{H}^{p}(\mathscr{L}, V)$ as the $p^{\text {th }}$ cohomology group of a certain subcomplex of the standard one. We define the $\mathbb{Z}$-gradation of $L \mathfrak{g} \oplus \mathfrak{D}$ by

$$
(L \mathfrak{g} \oplus \mathfrak{d})[m]:=\mathfrak{g} \otimes t^{m} \oplus \mathbb{C} t^{m+1} \frac{d}{d t} \quad \text { for } \quad m \in \mathbb{Z},
$$

and the $\mathbb{Z}$-gradation of $\hat{\mathcal{O}}$ by

$$
\hat{\mathcal{O}}[m]:=\hat{\mathcal{O}} \cap \hat{\mathscr{A}}[m] \text { for } m \in \mathbb{Z} .
$$

We shall mainly deal with $\tilde{H}^{\cdot}(L \mathfrak{g} \oplus \mathfrak{D}, \widehat{\mathcal{O}})$.

3.2. Let us recall the definition of the usual Lie algebra cohomology. For a Lie algebra $\mathscr{L}$ and an $\mathscr{L}$-module $V$, the group of $p$-cochains is defined by

$$
C^{p}=C^{p}(\mathscr{L}, V):=\operatorname{Hom}_{\mathbb{C}}\left(\wedge^{p} \mathscr{L}, V\right) \text { for } p \geqq 0 .
$$

We put $C^{p}:=0$ for $p<0$ in convention. The differential $d: C^{p} \rightarrow C^{p+1}$ is defined by

$$
\begin{aligned}
(d f)\left(l_{1}, \ldots, l_{p+1}\right):= & \sum_{1 \leqq i \leqq p+1}(-1)^{i-1} l_{i}\left(f\left(l_{1}, \ldots, \hat{l}_{i}, \ldots, l_{p+1}\right)\right) \\
& +\sum_{1 \leqq i<j \leqq p+1}(-1)^{i+j} f\left(\left[l_{i}, l_{j}\right], l_{1}, \ldots, \hat{l}_{i}, \ldots, \hat{l}_{j}, \ldots, l_{p+1}\right)
\end{aligned}
$$

for $f \in C^{p}$ and $l_{i} \in \mathscr{L}$, where the hats denote the eliminations of the arguments. The group of $p$-cocycles and that of $p$-coboundaries are defined by

$$
Z^{p}:=\operatorname{Ker}\left(d: C^{p} \rightarrow C^{p+1}\right) \quad \text { and } \quad B^{p}:=\operatorname{Im}\left(d: C^{p-1} \rightarrow C^{p}\right) .
$$

The $p^{\text {th }}$ cohomology group of $\mathscr{L}$ with coefficients in $V$ is defined by

$$
H^{p}(\mathscr{L}, V):=Z^{p} / B^{p} .
$$

3.3. Now let us introduce a cohomology $\tilde{H}^{\cdot}(\mathscr{L}, V)$. Assume that $\mathscr{L}=\bigoplus_{m \in \mathbb{Z}} \mathscr{L}[m]$ is a $\mathbb{Z}$-graded Lie algebra and $V=\bigoplus_{m \in \mathbb{Z}} V[m]$ is a $\mathbb{Z}$-graded $\mathscr{L}$-module. Let $M=\bigoplus_{m \in \mathbb{Z}} M[m]$ and $N=\bigoplus_{m \in \mathbb{Z}} N[m]$ be $\mathbb{Z}$-graded vector spaces. Then a linear map 
$f$ from $M$ to $N$ can be uniquely represented in the following form:

$$
f=\sum_{m \in \mathbb{Z}} f[m], \quad \text { where } f[m](M[n]) \subset N[m+n] .
$$

In this notation, we put

$$
\widetilde{\operatorname{Hom}}_{\mathbb{C}}(M, N):=\left\{f \in \operatorname{Hom}_{\mathbb{C}}(M, N) \mid f[m] \neq 0 \text { only for finitely many } m \text { 's }\right\} .
$$

We define the $\mathbb{Z}$-gradation of $\wedge^{p} \mathscr{L}$ by

$$
\left(\wedge^{p} \mathscr{L}\right)[m]:=\sum_{m_{1}+\cdots+m_{p}=m} \mathscr{L}\left[m_{1}\right] \wedge \cdots \wedge \mathscr{L}\left[m_{p}\right] \text { for } m \in \mathbb{Z} .
$$

The subgroup $\tilde{C}^{p}$ of $C^{p}$ is defined by

$$
\tilde{C}^{p}=\tilde{C}^{p}(\mathscr{L}, V):=\widetilde{\operatorname{Hom}}_{\mathbb{C}}\left(\wedge^{p} \mathscr{L}, V\right) .
$$

Since $(d f)[m]=d(f[m])$ for $f \in C^{p}$, the direct sum $\bigoplus_{m \in \mathbb{Z}} \tilde{C}^{p}$ is closed under the action of $d$. Thus we can define the cohomology $\tilde{H}^{\cdot}(\mathscr{L}, V)$ by

where we put

$$
\tilde{H}^{p}(\mathscr{L}, V):=\tilde{Z}^{p}(\mathscr{L}, V) / \tilde{B}^{p}(\mathscr{L}, V) \text { for } p \in \mathbb{Z},
$$

$$
\tilde{Z}^{p}(\mathscr{L}, V):=\operatorname{Ker}\left(d: \tilde{C}^{p} \rightarrow \tilde{C}^{p+1}\right) \quad \text { and } \quad \tilde{B}^{p}(\mathscr{L}, V):=\operatorname{Im}\left(d: \tilde{C}^{p-1} \rightarrow \tilde{C}^{p}\right) .
$$

Lemma 3.1. The 2-cocycle $\omega$ defined by (2.32) belongs to $\tilde{Z}^{2}(L \mathfrak{g} \oplus \mathrm{D}, \hat{\mathcal{O}})$.

For the proof, it suffices to see that $\omega$ belongs to $\widetilde{C}^{2}(L \mathfrak{g} \oplus, \hat{\mathcal{O}})$. But then this is obvious by Lemma 2.3 .

Regarding $\mathbb{C}$ as a $\mathbb{Z}$-graded vector space by

$$
\mathbb{C}[0]:=\mathbb{C} \text { and } \mathbb{C}[m]:=0 \text { for } m \neq 0 .
$$

we obtain the following.

Lemma 3.2. Let $\mathscr{S}$ be a $\mathbb{Z}$-graded subalgebra of $L \mathfrak{b}_{-} \oplus$ d. Put $\mathscr{L}:=L n_{+} \oplus \mathscr{S}$. Suppose that $\mathscr{L}$ is a subalgebra of $\mathrm{Lg} \oplus \mathrm{D}$. Then there is a canonical isomorphism from $\tilde{H}^{\cdot}(\mathscr{L}, \hat{\mathcal{O}})$ onto $H^{*}(\mathscr{S}, \mathbb{C})$. (In the proof, we can find the explicit form of this isomorphism.)

Proof. Step 1. In order to apply the theory of spectral sequences to the complex $\tilde{C}^{\bullet}(\mathscr{L}, \hat{\mathcal{O}})$, we shall introduce certain filtrations as follows. Define the increasing filtration of $\wedge^{n} \mathscr{L}$ by

$$
F_{a} \wedge^{n} \mathscr{L}:=\wedge^{a} \mathscr{L} \otimes \wedge^{n-a} \mathscr{S} \subset \wedge^{n} \mathscr{L} \text { for } a=0,1, \ldots, n .
$$

Put $\mathrm{Gr}_{a}\left(\wedge^{n} \mathscr{L}\right):=\left(F_{a} \wedge^{n} \mathscr{L}\right) /\left(F_{a-1} \wedge^{n} \mathscr{L}\right)$. Then we have

$$
\operatorname{Gr}_{a}\left(\wedge^{n} \mathscr{L}\right) \cong \wedge^{a}(\mathscr{L} / \mathscr{S}) \otimes \wedge^{n-a} \mathscr{S} \text { for } a=0,1, \ldots, n
$$

Define the $\mathbb{Z}$-gradation and the decreasing filtration of $\mathcal{O}$ by

$$
\begin{aligned}
G^{a} \mathcal{O} & :=\left(\sum_{\alpha \in \Delta_{+}} \sum_{m \in \mathbb{Z}} \mathbb{C} x_{\alpha}[m]\right)^{a} \subset \mathcal{O}, \\
F^{a} \mathcal{O} & :=\bigoplus_{i \geqq a} G^{a} \mathcal{O} \text { for } a \in \mathbb{N} .
\end{aligned}
$$


Then we have $F^{0} \mathcal{O}=\mathcal{O}$ and $F^{a} \mathcal{O}=G^{a} \mathcal{O} \mathscr{L} F^{a+1} \mathcal{O}$. Put

$$
G^{a} \hat{\mathcal{O}}:=\left(G^{a} \mathcal{O}\right) \hat{n}, \quad F^{a} \hat{\mathcal{O}}:=\left(F^{a} \mathcal{O}\right)^{\hat{n}}, \quad \text { and } \quad \stackrel{a}{\mathrm{Gr}} \hat{\mathcal{O}}:=F^{a} \hat{\mathcal{O}} / F^{a+1} \hat{\mathcal{O}}
$$

where ()$^{\wedge}$ denotes the closure in $\hat{\mathcal{O}}$. Then we have $F^{0} \hat{\mathcal{O}}=\hat{\mathcal{O}}$ and $F^{a} \hat{\mathcal{O}}=G^{a} \hat{\mathcal{O}} \oplus F^{a+1} \hat{\mathcal{O}}$, hence

$$
\mathrm{Gr}^{a} \hat{\mathcal{O}} \cong G^{a} \hat{\mathcal{O}} \text { as topological vector spaces. }
$$

In the following, we identify $\mathrm{Gr}^{a} \hat{\mathcal{O}}$ with $G^{a} \hat{\mathcal{O}}$. We remark that $\mathrm{Gr}^{0} \hat{\mathcal{O}} \cong G^{0} \hat{\mathcal{O}}=\mathbb{C}$. We define the decreasing filtration of $\widetilde{C}^{n}(\mathscr{L}, \hat{\mathcal{O}})$ by

$$
\begin{aligned}
F^{p, n-p} & :=F^{p} C^{n}(\mathscr{L}, \hat{\mathcal{O}}) \\
& :=\left\{f \in \tilde{C}^{n}(\mathscr{L}, \hat{\mathcal{O}}) \mid f\left(F_{a} \wedge^{n} \mathscr{L}\right) \subset F^{p-a} \hat{\mathcal{O}} \text { for } a=0,1, \ldots, n\right\} .
\end{aligned}
$$

It is obvious that this filtration is compatible with the differential: $d F^{p, n-p} \subset F^{p, n+1-p}$.

Step 2. Let us consider the spectral sequence $E_{a}^{p, q}$ attached to the filtration $F^{p, q}$. The $E_{0}$ terms shall be determined as follows. Putting

$$
E_{0}^{p, n-p}(a):=\widetilde{\operatorname{Hom}}_{\mathbb{C}}\left(\mathrm{Gr}_{a}\left(\wedge^{n} \mathscr{L}\right), \mathrm{Gr}^{p-a} \hat{\mathcal{O}}\right)
$$

we obtain

$$
E_{0}^{p, n-p}=F^{p, n-p} / F^{p+1, n-p-1} \cong \bigoplus_{a=0}^{p} E_{0}^{p, n-p}(a) .
$$

By definition of $\mathscr{L}$ and $\mathscr{S}$, we have

$$
\mathscr{L} \cdot F^{p-a} \hat{\mathcal{O}} \subset F^{p-a-1} \hat{\mathcal{O}} \text { and } \mathscr{S} \cdot F^{p-a} \hat{\mathcal{O}} \subset F^{p-a} \hat{\mathcal{O}}
$$

Thus we obtain the following induced maps:

$$
\begin{aligned}
& \sigma_{-1}(l): \mathrm{Gr}^{p-a} \hat{\mathcal{O}} \rightarrow \mathrm{Gr}^{p-a-1} \hat{\mathcal{O}} \text { for } l \in \mathscr{L}, \\
& \sigma_{0}(s): \mathrm{Gr}^{p-a} \hat{\mathcal{O}} \rightarrow \mathrm{Gr}^{p-a} \hat{\mathcal{O}} \quad \text { for } \quad s \in \mathscr{S} \text {. }
\end{aligned}
$$

For $l \in \mathscr{L}$, let $\bar{l}$ denote the class in $\mathscr{L} / \mathscr{S}$ represented by $l$. Then $\sigma_{-1}(l)$ depends only on $\bar{l} \in \mathscr{L} / \mathscr{S}$. In the above notation, the induced differential $d_{0}^{p}$ of $E_{0}^{p}$ is written in the following form:

$$
\begin{aligned}
d_{0}^{p} f\left(\bar{l}_{1}, \ldots, \bar{l}_{a}, s_{1}, \ldots, s_{n+1-a}\right) & \\
:= & \sum_{1 \leqq i \leqq a}(-1)^{i-1} \sigma_{-1}\left(l_{i}\right) f\left(\bar{l}_{1}, \ldots, \hat{\bar{l}}_{i}, \ldots, \bar{l}_{a}, s_{1}, \ldots, s_{n+1-a}\right) \\
& +\sum_{1 \leqq i \leqq n+1-a}(-1)^{i-1} \sigma_{0}\left(s_{i}\right) f\left(\bar{l}_{1}, \ldots, \bar{l}_{a} s_{1}, \ldots, \hat{s}_{i}, \ldots, s_{n+1-a}\right) \\
& +\sum_{\substack{1 \leqq i \leqq a \\
1 \leqq n+1-a}}(-1)^{i+j-1} f\left(\overline{\left[l_{i}, s_{j}\right]}, \bar{l}_{1}, \ldots, \hat{\bar{l}}_{i}, \ldots, \bar{l}_{a}, s_{1}, \ldots, \hat{s}_{j}, \ldots, s_{n+1-a}\right) \\
& +\sum_{1 \leqq i<j \leqq n+1-a}(-1)^{i+j+a} f\left(\bar{l}_{1}, \ldots, \bar{l}_{a},\left[s_{i}, s_{j}\right], s_{1}, \ldots, \hat{s}_{i}, \ldots, \hat{s}_{j}, \ldots, s_{n+1-a}\right),
\end{aligned}
$$

where $f \in E_{0}^{p, n-p}, l_{i} \in \mathscr{L}$ and $s_{j} \in \mathscr{S}$. 
Step 3. Let us calculate the $E_{1}$ terms. The group $E_{1}^{p, n-p}$ is the $n^{\text {th }}$ cohomology group of the complex $\left(E_{0}^{p, \cdot-p}, d_{0}^{p}\right)$. As a special case of the results in Step 1, we have obtained $E_{0}^{0, n} \cong \widetilde{\operatorname{Hom}}_{\mathbb{C}}\left(\wedge^{n} \mathscr{S}, \mathbb{C}\right)=\widetilde{C}^{n}(\mathscr{S}, \mathbb{C})$. This implies that

$$
E_{1}^{0, n}=\tilde{H}^{n}(\mathscr{S}, \mathbb{C}) \text { for } n \in \mathbb{Z} .
$$

In the following, we shall show that $E_{1}^{p, n}=0$ if $p \neq 0$. For this purpose, we introduce the linear map $\eta$ from $E_{0}^{p, n-p}$ to $E_{0}^{p, n-1-p}$ by

$$
\eta f\left(\bar{l}_{1}, \ldots, \bar{l}_{a-1}, s_{1}, \ldots, s_{n-a}\right):=\sum_{\substack{\alpha \in \Delta_{+} \\ m \in \mathbb{Z}}} x_{\alpha}[m] f\left(\overline{e_{\alpha} \otimes t^{-m}}, \bar{l}_{1}, \ldots, \bar{l}_{a-1}, s_{1}, \ldots, s_{n-a}\right),
$$

where $f \in E_{0}^{p, n-p}, l_{i} \in \mathscr{L}$ and $s_{j} \in \mathscr{S}$. In order to check the well-definedness of $\eta$, we have to prove that the right-hand side of (3.28) converges in $\mathrm{Gr}^{p-a+1} \hat{\mathcal{O}}=G^{p-a+1} \hat{\mathcal{O}}$. Because of the definition of Hom, we can suppose that $f=f[M]$ and $l_{1} \wedge \cdots \wedge$ $l_{a-1} \wedge s_{1} \wedge \cdots \wedge s_{n-a} \in\left(\wedge^{n-1} \mathscr{L}\right)[N]$ for some $M$ and $N$. Putting

we obtain

$$
\begin{aligned}
& X_{\alpha, m}:=f\left(\overline{e_{\alpha} \otimes t^{-m}}, \bar{l}_{1}, \ldots, \bar{l}_{a-1}, s_{1}, \ldots, s_{n-a}\right), \\
& \mu(m):=\max \{m, M+N-m\},
\end{aligned}
$$

$$
x_{\alpha}[m] X_{\alpha, m} \in G^{p-a+1} \hat{\mathcal{O}} \cap \hat{\mathscr{A}}^{\mu(m)}[M+N] .
$$

The subspace $G^{p-a+1} \hat{\mathcal{O}}$ is closed in $\hat{\mathscr{A}}$. Hence, by the definition of $\hat{\mathscr{A}}[M+N]$, it follows that the right-hand side of (3.28) converges in $G^{p-a+1} \hat{\mathcal{O}}$.

From Lemma 1.1 and the definition of $\tilde{\pi}$, we can write

$$
\sigma_{-1}\left(e_{\alpha} \otimes t^{-m}\right)=\frac{\partial}{\partial x_{\alpha}[m]} \text { for } \alpha \in \Delta_{+} \quad \text { and } m \in \mathbb{Z}
$$

Furthermore, we find that

$$
\left[\sigma_{-1}(l), \sigma_{0}(s)\right]=\sigma_{-1}([l, s]) \text { for } \quad l \in \mathscr{L} \quad \text { and } \quad s \in \mathscr{S} .
$$

We can deduce from (3.32) and (3.33) that, for $s \in \mathscr{S}$,

$$
\left.\sum_{\substack{\alpha \in \Delta_{+} \\ m \in \mathbb{Z}}}\left\{\left[\sigma_{0}(s), x_{\alpha}[m]\right] \otimes\left(\overline{e_{\alpha} \otimes t^{-m}}\right)+x_{\alpha}[m] \otimes \overline{\left[s, e_{\alpha} \otimes t^{-m}\right.}\right]\right\}=0 .
$$

Tedious calculations using (3.32) and (3.34) show that

$$
\eta d_{0}^{p}+d_{0}^{p} \eta=p \text { id } \quad \text { on } E_{0}^{p, \cdot-p} \text {. }
$$

Thus, if $p \neq 0$, then $p^{-1} \eta$ is a homotopy operator joining the identity map of $E_{1}^{p, \cdot-p}$ with the zero map. Consequently we find that

$$
E_{1}^{p, n-p}=0 \text { for } p \neq 0 \text { and } n \in \mathbb{Z} .
$$

Step 4. We obtain from (3.27) and (3.36) that

$$
\tilde{H}^{n}(\mathscr{L}, \widehat{\mathcal{O}}) \cong E_{\infty}^{0, n} \cong E_{1}^{0, n} \cong \tilde{H}^{n}(\mathscr{S}, \mathbb{C}) \text { for } n \in \mathbb{Z} .
$$

Lemma 3.3. $\tilde{H}^{1}\left(L \mathfrak{b}_{+} \oplus \mathfrak{D}, \widehat{\mathcal{O}}\right)=0$.

Proof. Applying Lemma 3.2 to the case $\mathscr{S}=L \mathfrak{h} \oplus \mathfrak{D}$, we obtain

$$
\tilde{H}^{1}(L \mathfrak{b}+\oplus \mathfrak{d}, \hat{\mathcal{O}}) \cong \tilde{H}^{1}(\mathscr{S}, \mathbb{C}) \cong \tilde{Z}^{1}(\mathscr{S}, \mathbb{C}) .
$$

But it is obvious that $[\mathscr{S}, \mathscr{S}]=\mathscr{S}$, hence $\tilde{Z}^{1}(\mathscr{S}, \mathbb{C})=0$. 
Lemma 3.4. $H^{0}\left(L n_{+}, \hat{\mathcal{O}}\right)=\mathbb{C}$.

Proof. An application of Lemma 3.2 to the case $\mathscr{S}=0$ yields that

$$
\tilde{H}^{0}\left(L n_{+}, \hat{\mathcal{O}}\right) \cong \tilde{H}^{0}(0, \mathbb{C})=\widetilde{\operatorname{Hom}}_{\mathbb{C}}(\mathbb{C}, \mathbb{C})=\mathbb{C} \text {. }
$$

But then it is easy to see that $\tilde{H}^{0}\left(L n_{+}, \widehat{\mathcal{O}}\right)=H^{0}\left(L n_{+}, \widehat{\mathcal{O}}\right)$.

3.4. In the next section, we shall need the following lemma.

Lemma 3.5. The inclusion map from $L \mathfrak{h} \oplus \mathfrak{D}$ into $L \mathfrak{b} \_\oplus \mathfrak{D}$ induces the isomorphism from $\widetilde{H}^{p}(L \mathfrak{b}-\oplus d, \mathbb{C})$ onto $\widetilde{H}^{p}(L \mathfrak{h} \oplus \grave{d}, \mathbb{C})$.

This is the special case of the following lemma.

Lemma 3.6. Let $\mathscr{L}$ be a $\mathbb{Z}$-graded Lie algebra and $h$ an element of $\mathscr{L}$. Suppose that the adjoint action of $h$ on $\mathscr{L}$ is diagonalizable. Put $\mathscr{L}_{a}:=\{l \in \mathscr{L} \mid[h, l]=$ al $\}$ for $a \in \mathbb{C}$. Define the $\mathbb{C}$-gradation of $\wedge^{p} \mathscr{L}$ and that of $\tilde{C}^{p}=\tilde{C}^{p}(\mathscr{L}, \mathbb{C})$ by

$$
\begin{aligned}
\left(\wedge^{p} \mathscr{L}\right)_{a} & :=\sum_{a_{1}+\cdots+a_{p}=a} \mathscr{L}_{a_{1}} \wedge \cdots \wedge \mathscr{L}_{a_{p}}, \\
\left(\tilde{C}^{p}\right)_{a} & :=\left\{f \in \tilde{C}^{p} \mid f\left(\left(\wedge^{p} \mathscr{L}\right)_{b}\right)=0 \text { for } b \neq a\right\} \text { for } a \in \mathbb{C} .
\end{aligned}
$$

Then $\left(\tilde{C}^{*}\right)_{a}$ is a subcomplex of $\tilde{C}^{\cdot}$. Furthermore $\tilde{H}^{p}(\mathscr{L}, \mathbb{C})$ is isomorphic to the $p^{\text {th }}$ cohomology group of $\left(\widetilde{C}^{\bullet}\right)_{0}$.

Proof. Since the first assertion is obvious, it suffices to show the second assertion. We can define the linear map $i(h)$ from $\widetilde{C}^{p}$ to $\widetilde{C}^{p-1}$ by

$$
(i(h) f)\left(l_{1}, \ldots, l_{p-1}\right):=f\left(h, l_{1}, \ldots, l_{p-1}\right) \text { for } l_{i} \in \mathscr{L} .
$$

Then $i(h)\left(\tilde{C}^{p}\right)_{a} \subset\left(\tilde{C}^{p-1}\right)_{a}$ for $a \in \mathbb{C}$. Moreover a straightforward computation shows that $i(h) d+d i(h)=a$ id on $\left(\tilde{C}^{\bullet}\right)_{a}$ for $a \in \mathbb{C}$. Hence, if $a \neq 0$, then $a^{-1} i(h)$ is a homotopy operator joining the identity mapping of $\left(\tilde{C}^{*}\right)_{a}$ with the zero map. It follows that $H^{p}\left(\left(\widetilde{C}^{\bullet}\right)_{a}\right)=0$ for $a \neq 0$. Hence we conclude that

$$
\tilde{H}^{p}(\mathscr{L}, \mathbb{C})=H^{p}\left(\tilde{C}^{\bullet}\right)=\bigoplus_{a \in \mathbb{C}} H^{p}\left(\left(\tilde{C}^{\bullet}\right)_{a}\right)=H^{p}\left(\left(\tilde{C}^{\bullet}\right)_{0}\right)
$$

\section{Fock Space Representations of Affine Lie Algebras}

4.1. The results of the previous section allow us to prove the following theorem, which is a starting point of the theory of Fock space representations of the affine algebras.

Theorem 4.1. There is a unique element $\Gamma$ of $\tilde{C}^{1}(L \mathfrak{g} \oplus \mathcal{D}, \hat{\mathcal{O}})$ satisfying the following properties:

$$
\begin{array}{cl}
d \Gamma=c_{2}-\omega & \text { in } \tilde{B}^{2}(L \mathfrak{g} \oplus \mathfrak{D}, \hat{\mathcal{O}}), \\
\Gamma=0 & \text { on } L \mathfrak{b}_{+} \oplus \mathfrak{D} .
\end{array}
$$

Proof. Existence. From Lemma 2.4 and 3.1, it follows that $\omega$ and $c_{2}$ define the same cohomology class in $\tilde{H}^{2}(L \mathfrak{h} \oplus \mathcal{D}, \mathbb{C})$. On the other hand, Lemmas 3.2 and 3.5 yield the following isomorphisms:

$$
\tilde{H}^{2}(L \mathfrak{g} \oplus \mathrm{d}, \hat{\mathcal{O}}) \cong \tilde{H}^{2}\left(L \mathfrak{b}_{-} \oplus \mathrm{d}, \mathbb{C}\right) \cong \tilde{H}^{2}(L \mathfrak{h} \oplus \mathfrak{D}, \mathbb{C})
$$


Hence there is an element $\tilde{\Gamma}$ of $\tilde{C}^{1}(L \mathfrak{g} \oplus \mathfrak{d}, \hat{\mathcal{O}})$ such that $d \tilde{\Gamma}=c_{2}-\omega$ in $\widetilde{B}^{2}(L \mathfrak{g} \oplus \mathfrak{d}, \hat{\mathcal{O}})$. Because of Lemma 2.4, it is found that $d \tilde{\Gamma}=0$ on $\wedge^{2}\left(L b_{+} \oplus D\right)$. Thus, by Lemma 3.3, it is possible to find $a \in \hat{\mathcal{O}}=\widetilde{C}^{0}\left(L \mathfrak{b}_{+} \oplus \mathfrak{d}, \mathbb{C}\right)$ so that $\tilde{\Gamma}=d a$ on $L \mathfrak{b}_{+} \oplus \mathcal{D}$. Put

$$
\Gamma:=\tilde{\Gamma}-d a \in \tilde{C}^{1}(L \mathfrak{g} \oplus \mathfrak{D}, \hat{\mathcal{O}}) .
$$

Then it is obvious that $\Gamma$ satisfies (4.1) and (4.2).

Uniqueness. Suppose that $\Gamma^{\prime}$ is an element of $\tilde{C}^{1}(L \mathfrak{g} \oplus \mathfrak{d}, \hat{\mathcal{O}})$ satisfying the conditions similar to (4.1) and (4.2). Put $u:=\Gamma^{\prime}-\Gamma$. Then $d u=0$ in $\widetilde{B}^{2}(L \mathfrak{g} \oplus \mathrm{D}, \hat{\mathcal{O}})$ and $u=0$ on $L \mathfrak{b}_{+} \oplus \mathfrak{D}$. By Lemma 3.3, it is possible to choose $b \in \hat{\mathcal{O}}$ so that $u=d b$ on $L \mathfrak{b}_{+} \oplus \mathfrak{D}$. Hence, from Lemma 3.4, it follows that $b$ is in $\mathbb{C}$. Thus $u=d b=0$.

Definition. In the notation in Theorem 4.1, we can define the Lie algebra homomorphism $\pi$ from the affine-Virasoro algebra $\hat{\mathfrak{g}} \oplus \mathrm{Vir}$ to $\hat{\mathscr{A}}$ by

$$
\begin{aligned}
& \pi(X \otimes f):=\tilde{\pi}(X \otimes f)+\Gamma(X \otimes f) \quad \text { for } X \in \mathrm{g} \text { and } f \in \mathbb{C}\left[t, t^{-1}\right], \\
& \pi\left(f \frac{d}{d t}\right):=\tilde{\pi}\left(f \frac{d}{d t}\right) \quad \text { for } \quad f \in \mathbb{C}\left[t, t^{-1}\right], \\
& \pi(K):=k=\kappa-g^{*} \text { and } \pi(C):=c=k \operatorname{dim} \mathfrak{g} / \kappa .
\end{aligned}
$$

We call $\Gamma$ the correction for currents. (For the definition of $\tilde{\pi}$, see (2.21) and (2.22).) Recall that $\hat{\mathscr{A}}$ acts on the Fock space $\mathscr{F}_{\lambda}$, where $\lambda \in \mathfrak{h}^{*}$. Thus we obtain a representation of $\hat{\mathfrak{g}} \oplus$ Vir on $\mathscr{F}_{\lambda}$, which is called the Fock space representation of the affine-Virasoro algebra.

It is easy to see that the vector $|\lambda\rangle \in \mathscr{F}_{\lambda}$ satisfies the highest weight condition (0.11) for $\hat{\mathfrak{g}} \oplus$ Vir. For $X \in \mathfrak{g}$ and $m \in \mathbb{Z}$, put $X[m]:=\pi\left(X \otimes t^{m}\right)$ and $L_{m}:=\pi\left(-t^{m+1} \frac{d}{d t}\right)$. Formally we define the current operator $X(z)$ for $X \in \mathfrak{g}$ by $(0.9)$.

4.2. Let us determine the explicit form of $\Gamma$. The condition (4.1) is equivalent to the following:

$$
\Gamma\left(\left[l_{1}, l_{2}\right]\right)=\omega\left(l_{1}, l_{2}\right)-c_{2}\left(l_{1}, l_{2}\right)+l_{1} \cdot \Gamma\left(l_{2}\right)-l_{2} \cdot \Gamma\left(l_{1}\right) \quad \text { for } \quad l_{1}, l_{2} \in L \mathfrak{g} \oplus \mathfrak{d} .
$$

Hence, because of (4.2), the correction $\Gamma$ is uniquely determined from the set $\left\{\Gamma\left(F_{i} \otimes t^{m}\right) \mid i=1, \ldots, r\right.$ and $\left.m \in \mathbb{Z}\right\}$.

Proposition 4.2. There is a unique set $\left\{\gamma_{i}\right\}_{i=1}^{r}$ of complex numbers such that

$$
\Gamma_{i}\left(F_{i} \otimes t^{m}\right)=-\gamma_{i} m x_{\alpha_{1}}[m] \text { for } i=1, \ldots, r \text { and } m \in \mathbb{Z} .
$$

Equivalently we have

$$
F_{i}(z)=\tilde{F}_{i}(z)+\gamma_{i} \partial x_{\alpha_{i}}(z) \text { for } i=1, \ldots, r .
$$

Proof. We fix $i=1, \ldots, r$ in the following. Put $\Gamma_{i}[m]:=\Gamma\left(F_{i} \otimes t^{m}\right)$ for $m \in \mathbb{Z}$ and $\Gamma_{i}(z):=\sum_{m \in \mathbb{Z}} z^{-m-1} \Gamma_{i}[m]$. Let $H$ be in $\mathfrak{h}$. Then Lemmas 2.3 and 2.5 imply the following OPE:

$$
H(z) \Gamma_{i}(z) \sim \frac{-\left(\alpha_{i} \mid H\right) \Gamma_{i}(w)}{z-w}+\frac{a(w)}{(z-w)^{2}},
$$


where $a(w)$ is some formal Laurent series with coefficients in $\hat{\mathcal{O}}$. This implies that

$$
\left[H[0], \Gamma_{i}[n]\right]=-\left(\alpha_{i} \mid H\right) \Gamma_{i}[n] \text { for } H \in \mathfrak{b} \text { and } n \in \mathbb{Z} \text {. }
$$

In addition, Lemma 2.3 admits us to obtain the following OPE:

$$
T(z) \Gamma_{i}(z) \sim \frac{\partial \Gamma_{i}(w)}{z-w}+\frac{\Gamma_{i}(w)}{(z-w)^{2}}+\frac{b(w)}{(z-w)^{3}},
$$

where we put $b(w):=\Omega_{2}\left(F_{i} ; w\right)$. Denote the expansion of $b(w)$ by $\sum_{m \in \mathbb{Z}} z^{-m} b_{m}$. Then (4.13) is equivalent to the following commutation relations:

$$
\left[L_{m}, \Gamma_{i}[[n]]=-n \Gamma_{i}[m+n]+\frac{(m+1) m}{2} b_{m+n} \text { for } m, n \in \mathbb{Z}\right.
$$

As a special case of this, we obtain

$$
\left[L_{0}, \Gamma_{i}[n]\right]=-n \Gamma_{i}[n] \text { for } n \in \mathbb{Z} .
$$

From (4.13) and (4.16), it is found that $\Gamma[n]$ can be written in the following form:

$$
\Gamma_{i}[n]=a_{n} x_{\alpha_{i}}[n] \text { for } n \in \mathbb{Z},
$$

where $\left\{a_{n}\right\}_{n \in \mathbb{Z}}$ is some set of complex numbers. Using this, rewrite (4.15) in the following form:

$$
\frac{(m+1) m}{2} b_{m+n}=\left(n a_{m+n}-(m+n) a_{n}\right) x_{\alpha_{i}}[m+n] \text { for } m, n \in \mathbb{Z} .
$$

Substituting $m=-1$ into (4.18), we obtain $(n-1) a_{n}=n a_{n-1}$ for $n \in \mathbb{Z}$. Hence the case for $m=1$ implies $b_{n+1}=0$ for $n \in \mathbb{Z}$. Furthermore, by the case for $m=-2$ and $n=1$, we obtain $a_{1}=-a_{-1}$. Thus we conclude that $a_{n}=-n a_{-1}$ for $n \in \mathbb{Z}$. Putting $\gamma_{i}:=a_{-1}$, we finish the proof.

Remark 4.3. For $i=1, \ldots, r$, the constant $\gamma_{i}$ can be determined by the OPE of $E_{i}(z)$ and $F_{i}(z)$. The result is the following. Define the constants $\left\{N_{\alpha, \beta}\right\}$ by

$$
\left[e_{\alpha}, e_{\beta}\right]=N_{\alpha, \beta} e_{\alpha+\beta}, \quad \text { where } \alpha, \beta \text { and } \alpha+\beta \text { are in } \Delta \text {. }
$$

For convenience, we put $N_{\alpha, \beta}:=0$ unless $\alpha, \beta$ and $\alpha+\beta$ are roots of $g$. Recall that positive roots have been numbered by a fixed order: $\Delta_{+}=\left\{\beta_{1}, \ldots, \beta_{s}\right\}$. We define the total order $\prec$ in $\Delta_{+}$by

$$
\beta_{1} \prec \beta_{2} \prec \cdots \prec \beta_{s} .
$$

In this notation, for $i=1, \ldots, r$, the constant $\gamma_{i}$ can be written in the following form:

$$
\gamma_{i}=\frac{2 k}{\left(\alpha_{i} \mid \alpha_{i}\right)}+\sum_{\substack{\alpha \in \Delta_{+} \\ \alpha>\alpha_{i}}} N_{\alpha, \alpha_{i}} N_{\alpha+\alpha_{i},-\alpha_{i}}
$$

4.3. For $\lambda \in \mathfrak{h}^{*}$, the Fock space $\mathscr{F}_{\lambda}$ decomposes into the weight subspaces. Putting, for $d \in \mathbb{N}$ and $\mu \in \mathfrak{h}^{*}$,

$$
\begin{aligned}
\mathscr{F}_{\lambda}[d] & :=\left\{v \in \mathscr{F}_{\lambda} \mid L_{0} v=\left(\Delta_{\lambda}+d\right) v\right\}, \\
\mathscr{F}_{\lambda}[d, \mu] & :=\left\{v \in \mathscr{F}_{\lambda}[d] \mid H[0] v=(\mu \mid H) v \text { for } H \in \mathfrak{h}\right\},
\end{aligned}
$$


we obtain $\mathscr{F}_{\lambda}=\bigoplus_{d=0}^{\infty} \mathscr{F}_{\lambda}[d]$ and $\mathscr{F}_{\lambda}[d]=\bigoplus_{\mu \in h^{*}} \mathscr{F}_{\lambda}[d, \mu]$. We remark that $\mathscr{F}_{\lambda}[d, \mu]$ is finite dimensional. Define the dual Fock space $\mathscr{F}_{\lambda}^{\dagger}$ by

where we put

$$
\mathscr{F}_{\lambda}^{\dagger}:=\bigoplus_{d=0}^{\infty} \mathscr{F}_{\lambda}^{\dagger}[d]
$$

$$
\mathscr{F}_{\lambda}^{\dagger}[d, \mu]:=\operatorname{Hom}_{\mathbb{C}}\left(\mathscr{F}_{\lambda}[d, \mu], \mathbb{C}\right), \quad \mathscr{F}_{\lambda}^{\dagger}[d]:=\bigoplus_{\mu \in h^{*}} \mathscr{F}_{\lambda}^{\dagger}[d, \mu] .
$$

Then $\mathscr{F}_{\lambda}^{\dagger}$ possesses the natural right $\hat{\mathfrak{g}} \oplus$ Vir-module structure. Denote by $\langle 1\rangle$ the natural pairing of $\mathscr{F}_{\lambda}^{\dagger}$ and $\mathscr{F}_{\lambda}$. Note that $\mathscr{F}_{\lambda}[0]$ is a $\mathfrak{g}$-submodule of $\mathscr{F}_{\lambda}$ and spanned by $\left\{\prod_{\beta \in \Delta_{+}} x_{\beta}[0]^{I_{\beta}} \mid\left(I_{\beta}\right)_{\beta \in \Delta_{+}} \in \mathbb{N}^{\Delta_{+}}\right\}$. Using this and the definition of $\pi$, we can prove the following.

Proposition 4.4. As g-modules, $\mathscr{F}_{\lambda}[0]$ and $\mathscr{F}_{\lambda}^{\dagger}[0]$ are isomorphic to $M_{\lambda}^{*}$ and $M_{\lambda}^{\dagger}$, respectively.

4.4. Let $\left\{J^{p}\right\}_{p=1}^{\operatorname{dim} g}$ be an orthonormal basis of $\mathfrak{g}$ with respect to the Killing form. For $p=1, \ldots, \operatorname{dim} g$ and $m, n \in \mathbb{Z}$, put

$$
{ }_{\circ}^{\circ} J^{p}[m] J^{p}[n]_{\circ}^{\circ}:=\left\{\begin{array}{lll}
J^{p}[m] J^{p}[n] & \text { if } & m \leqq n, \\
J^{p}[n] J^{p}[m] & \text { if } & m>n .
\end{array}\right.
$$

The Sugawara energy-momentum tensor $T^{\mathrm{SUG}}(z)$ is defined by $(0.12)$. Then, expanding $T^{\text {SUG }}(z)$ in the form $\sum_{m \in \mathbb{Z}} z^{-m-2} L_{m}^{\text {SUG }}$, we obtain a set $\left\{L_{m}^{\text {SUG }}\right\}_{m \in \mathbb{Z}}$ of operators acting on the Fock spaces. Let $\pi^{\prime}$ be the linear map from $\hat{\mathfrak{g}} \oplus \operatorname{Vir}$ to End $_{\mathbb{C}} \mathscr{F}_{\lambda}$ given by

$$
X \otimes t^{m} \mapsto X[m], \quad t^{m+1} \frac{d}{d t} \mapsto-L_{m}^{\mathrm{SUG}}, \quad K \mapsto k \mathrm{id}, \quad C \mapsto c \mathrm{id},
$$

where $X \in \mathfrak{g}$ and $m \in \mathbb{Z}$. Then $\pi^{\prime}$ is a representation of $\hat{\mathfrak{g}} \oplus$ Vir on $\mathscr{F}_{\lambda}$. The vector $|\lambda\rangle \in \mathscr{F}_{\lambda}$ satisfies

$$
L_{0}^{\mathrm{SUG}}|\lambda\rangle=\Delta_{\lambda}|\lambda\rangle \text { and } L_{m}^{\mathrm{SUG}}|\lambda\rangle=0 \text { for } m>0 .
$$

Theorem 4.5. For every $\kappa \neq 0, \lambda \in \mathfrak{h}^{*}$ and $m \in \mathbb{Z}$, we have

$$
L_{m}=L_{m}^{\mathrm{SUG}} \text { as operators acting on } \mathscr{F}_{\lambda} \text {. }
$$

Proof. For $\lambda \in \mathfrak{h}^{*}$, the algebra automorphism $\tau_{\lambda}$ of $\hat{\mathscr{A}}$ is uniquely characterized by the conditions

$$
\begin{aligned}
& \tau_{\lambda}\left(p_{i}[m]\right):=p_{i}[m]+\left(\lambda \mid H_{i}\right) \delta_{m, 0}, \\
& \tau_{\lambda}\left(x_{\alpha}[m]\right)=x_{\alpha}[m], \quad \tau_{\lambda}\left(\delta_{\alpha}[m]\right)=\delta_{\alpha}[m],
\end{aligned}
$$

where $m \in \mathbb{Z}, \alpha \in \Delta_{+}$and $i=1, \ldots, r$. Then the representation of $\hat{\mathfrak{g}} \oplus$ Vir on $\mathscr{F}_{0}$ given by the composition $\tau_{\lambda}{ }^{\circ} \pi$ is isomorphic to that on $\mathscr{F}_{\lambda}$ given by $\pi$. For $m, n \in \mathbb{Z}$ and $\alpha, \beta \in \Delta$, the operator $\pi_{\lambda}\left(e_{\alpha} \otimes t^{m}\right)$ polynomially depends on $\lambda$ as a linear map from $\mathscr{F}_{0}[n, \beta]$ to $\mathscr{F}_{0}[-m+n, \alpha+\beta]$. Similarly, for $m, n \in \mathbb{Z}$ and $\alpha \in \Delta$, the operator 
$\pi_{\lambda}\left(t^{m+1} \frac{d}{d t}\right)$ polynomially depends on $\lambda$ as a linear map from $\mathscr{F}_{0}[n, \alpha]$ to $\mathscr{F}_{0}[-m+n, \alpha]$. Hence, for the proof of the theorem, it suffices to show (4.28) for generic $\lambda$ 's. It is easy to see that the character of $\mathscr{F}_{\lambda}$ is equal to that of the Verma module $\mathscr{M}_{k, \lambda}$ of $\hat{\mathrm{g}}$ with level $k=\kappa-g^{*}$ and highest weight $\lambda$. Hence, for a generic $\lambda \in \mathfrak{h}^{*}$, the Verma module $\mathscr{M}_{k, \lambda}$ is irreducible and isomorphic to $\mathscr{F}_{\lambda}$. It suffices to prove the theorem for such a $\lambda$. It is obvious that $\left[L_{m}-L_{m}^{\text {sUG }}, X[n]\right]=0$ for $X \in \mathfrak{g}$ and $m, n \in \mathbb{Z}$. By the definition of $L_{m}^{\text {SUG }}$, it follows that $\left[L_{m}-L_{m}^{\text {SUG }}, L_{n}^{\text {SUG }}\right]=0$ for $m, n \in \mathbb{Z}$. This implies that $\left[L_{m}-L_{m}^{\mathrm{SUG}}, L_{n}-L_{n}^{\mathrm{SUG}}\right]=\left[L_{m}, L_{n}\right]-\left[L_{m}^{\mathrm{SUG}}, L_{n}^{\mathrm{SUG}}\right]$ for $m, n \in \mathbb{Z}$. Hence the representation of Vir on $\mathscr{F}_{\lambda}$ with central charge 0 can be defined by $-t^{m+1} \frac{d}{d t} \mapsto L_{m}-L_{m}^{\text {sug }}$ for $m \in \mathbb{Z}$. But the Schur lemma implies that $L_{m}-L_{m}^{\text {SUG }}=a_{m}$ id as operators acting on $\mathscr{F}_{\lambda}$ for some $a_{m} \in \mathbb{C}$. Thus, a bit computation shows that $L_{m}-L_{m}^{\text {SUG }}=0$ for $m \in \mathbb{Z}$.

\section{Screening Operators and Integral Representations}

5.1. In the previous section, we have defined the Fock space representations of the affine-Virasoro algebra and determined the explicit form of the correction for currents. Additionally, we have proved that the energy-momentum tensor written by free bosons are equal to the Sugawara one on the Fock spaces. In this section, we shall construct screening operators. Consequently, we shall obtain integral representations of correlation functions in the WZW model.

5.2. Let $\lambda$ and $\mu$ be in $\mathfrak{h}^{*}$. The linear isomorphism $e^{q[\lambda]}$ from $\mathscr{F}_{\mu}$ onto $\mathscr{F}_{\lambda+\mu}$ is uniquely characterized by the condition (0.14). Formally we put $z^{p[H ; 0]} v=z^{\left(\left.\lambda\right|_{H)}\right.} v$ for $v \in \mathscr{F}_{\lambda}$ and $H \in \mathfrak{h}$. We extend the normal product : : to them by

$$
: a_{-} e^{q[\lambda]} z^{p[\lambda ; 0]} a_{0} a_{+}::=a_{-} e^{q[\lambda]} z^{p[\lambda ; 0]} a_{0} a_{+} \text {for } a_{0} \in \mathscr{A}_{0} \text { and } a_{ \pm} \in \mathscr{A}_{ \pm} \text {. }
$$

Then the bosonic vertex operator $V(\lambda ; z)$ defined by $(0.15)$ is formally written in the form

where we put

$$
V(\lambda ; z)=: e^{q(\lambda ; z)} \cdot
$$

$$
q(\lambda ; z):=q[\lambda]+\frac{1}{\kappa}\left\{p[\lambda ; 0] \log z+\sum_{m \neq 0} \frac{z^{-m}}{-m} p[\lambda ; z]\right\} .
$$

We have the following OPE's:

$$
\begin{gathered}
p(H ; z) V(\lambda ; w) \sim \frac{(\lambda \mid H)}{z-w} V(\lambda ; w) \text { for } H \in \mathfrak{h}, \\
T(z) V(\lambda ; w) \sim \frac{\partial V(\lambda ; w)}{z-w}+\frac{\Delta_{\lambda} V(\lambda ; w)}{(z-w)^{2}} .
\end{gathered}
$$

Recall that, for $Y \in \mathfrak{n}_{+}$and $\alpha \in \Delta_{+}$, the polynomial $S_{\alpha}(Y ; x)$ in $x=\left(x_{\alpha}\right)_{\alpha \in \Delta_{+}}$has been defined by (1.10). For $i=1, \ldots, r$, put

$$
S_{i}(z):=\sum_{\alpha \in \Delta_{+}}: S_{\alpha}\left(E_{i} ; x(z)\right) \delta_{\alpha}(z)^{\circ}
$$




$$
\begin{aligned}
& V_{i}(z):=V\left(-\alpha_{i} ; z\right)=\dot{e} e^{q\left(-\alpha_{i} ; z\right)} \cdot \\
& s_{i}(z):=S_{i}(z) V_{i}(z)
\end{aligned}
$$

where we use the notation $x(z)=\left(x_{\alpha}(z)\right)_{\alpha \in A_{+}+}$. We remark that $S_{i}(z)$ commutes with $V_{i}(w)$. If we expand $s_{i}(z)$ in the form $e^{q[\lambda]} \sum_{m \in \mathbb{Z}} s_{i}[m] z^{-m+\tilde{p}[\lambda, 0]}$, then $s_{i}[m]$ is well-
defined as an element of $\hat{\mathscr{A}}$.

Theorem 5.1. For $i=1, \ldots, r$, the operator $s_{i}(z)$ satisfies the following OPE's:

$$
\begin{aligned}
& T(z) s_{i}(w) \sim \frac{\partial}{\partial w}\left\{\frac{s_{i}(w)}{z-w}\right\}, \\
& X(z) s_{i}(w) \sim 0 \text { for } X \in \mathfrak{b}_{+}, \\
& F_{j}(z) s_{i}(w) \sim-\kappa \delta_{i, j} \frac{\partial}{\partial w}\left\{\frac{V_{i}(w)}{z-w}\right\} \text { for } j=1, \ldots, r .
\end{aligned}
$$

(These OPE's are equivalent to the commutation relations (0.17).)

Definition. We call $\left\{s_{i}(z)\right\}_{i=1}^{r}$ the set of screening operators.

Proof of Theorem 5.1. Since $\Delta_{\alpha_{i}}=0$, it follows from (5.5) that $T(z) V_{i}(z) \sim \frac{\partial V_{i}(w)}{z-w}$. Lemma 1.1 and the Wick theorem (Lemma 2.1) yield that

$$
T(z) S_{i}(w) \sim \frac{\partial S_{i}(w)}{z-w}+\frac{S_{i}(w)}{(z-w)^{2}} .
$$

Therefore we obtain (5.9-1).

For $X \in n_{+}$, the OPE (5.9-2) follows from Lemmas 1.1, 1.4 and the Wick theorem. Let $H$ be in $\mathfrak{h}$. Using Lemmas 1.1, 1.2, 1.4 and the Wick theorem, we can obtain $H(z) S_{i}(w) \sim \frac{\left(\alpha_{i} \mid H\right) S_{i}(w)}{z-w}$. Lemma 1.2 and the OPE (5.4) imply that

$$
H(z) V_{i}(w) \sim p(H ; z) V_{i}(w) \sim-\frac{\left(\alpha_{i} \mid H\right) V_{i}(w)}{z-w}
$$

Thus we obtain the OPE (5.9-2) for $X=H$.

Using Lemma 1.4 and the Wick theorem, we can show the following OPE:

$$
\begin{aligned}
F_{j}(z) s_{i}(w) & \sim \frac{\delta_{i, j} \cdot p_{i}(w) V_{i}(w)^{\bullet}+A(w) V_{i}(w)}{z-w}+\frac{B(w) V_{i}(w)}{(z-w)^{2}} \\
& =\frac{-\kappa \delta_{i, j} \partial V_{i}(w)+A(w) V_{i}(w)}{z-w}+\frac{B(w) V_{i}(w)}{(z-w)^{2}},
\end{aligned}
$$

where $A(z)=\sum_{m \in \mathbb{Z}} z^{-m} A_{m}$ and $B(z)=\sum_{m \in \mathbb{Z}} z^{-m} B_{m}$ for some $A_{m}, B_{m} \in \hat{\mathcal{O}}$. This is equivalent to the following commutation relations:

$$
\left[F_{j}[m], s_{i}(w)\right]=-\kappa \delta_{i, j} w^{m} \partial V_{i}(w)+w^{m} A(w) V_{i}(w)+m w^{m-1} B(w) V_{i}(w),
$$

for $m \in \mathbb{Z}$. Take an element $E$ of $n_{+}$and an integer $n$. Then $E[n]$ commutes with $s_{i}(w)$ by (5.9-2). Hence, calculations of the commutators of $E[n]$ and both sides of 
(5.13) show that

$$
w^{m}[E[n], A(w)]+m w^{m-1}[E[n], B(w)]=0 .
$$

The case of $m=0$ implies $[E[n], A(w)]=0$. Hence we also obtain $[E[n], B(w)]=0$. Thus it follows from Lemma 3.4 that

$$
A_{m} \in \mathbb{C} \text { and } B_{m} \in \mathbb{C} \text { for } m \in \mathbb{Z} \text {. }
$$

Compute the commutators of $L_{n}$ and the both sides of (5.14). Then, owing to (5.9-1) and (5.15), we find

$$
w^{m+n+1} \partial A(w)+(n+1) w^{m+n} A(w)+m w^{m+n} \partial B(w)=0 .
$$

Considering the special cases for $(m, n)=(0,-1),(0,0)$ and $(1,-1)$ in order, we successively obtain $\partial A(w)=0, A(w)=0$ and $\partial B(w)=0$. Thus (5.13) is rewritten in the form

$$
\left[F_{j}[n], s_{i}(w)\right]=-\kappa \delta_{i, j} w^{m} \partial V_{i}(w)+B_{0} m w^{m-1} V_{i}(w)
$$

Let $H$ be in $\mathfrak{h}$. Then (5.11) implies that

$$
\left[H[n], V_{i}(w)\right]=-\left(\alpha_{j} \mid H\right) w^{n} V_{i}(w) .
$$

Using (5.15) and (5.18), compute the commutators of $H[n]$ and the both sides of (5.17). Then we obtain

$$
B_{0}\left(\alpha_{i} \mid H\right) n w^{n+m-1}=-\kappa \delta_{i, j}\left(\alpha_{j} \mid H\right) n w^{n+m-1} .
$$

Since we can assume $\left(\alpha_{i} \mid H\right) \neq 0$, we obtain $B_{0}=-\kappa \delta_{i, j}$. Therefore (5.17) becomes

$$
\left[F_{j}[m], s_{i}(w)\right]=-\kappa \delta_{i, j} \frac{\partial}{\partial w}\left\{w^{m} V_{i}(w)\right\} \text { for } m \in \mathbb{Z}
$$

which is equivalent to the OPE (5.9-3).

Definition. Expand $S_{i}(z)$ in the form $\sum_{m \in \mathbb{Z}} z^{-m-1} S_{i}[m]$, where $S_{i}[m] \in \hat{\mathscr{A}}$. For $i=1, \ldots, r$, define the vector $s_{i}$ in $\mathscr{F}_{-\alpha_{i}}$ by

$$
s_{i}:=S_{i}[-1]\left|-\alpha_{i}\right\rangle=\left[s_{i}(z)|0\rangle\right]_{z=0}
$$

We call $\left\{s_{i}\right\}_{i=1}^{r}$ the set of screening vectors.

Corollary 5.2. For $i=1, \ldots, r$, the screening vector $s_{i}$ has the following properties:

$$
\begin{aligned}
L_{0} s_{i} & =s_{i} \quad \text { and } L_{m} s_{i}=0 \text { for } m>0, \\
X[m] s_{i} & =0 \text { for } X \in \mathfrak{b}_{+} \quad \text { and } m \geqq 0, \\
F_{j}[0] s_{i} & \left.=-\kappa \delta_{i, j} L_{-1}\left|-\alpha_{i}>, \quad F_{j}[1] s_{i}=-\kappa \delta_{i, j}\right|-\alpha_{i}\right\rangle, \\
F_{j}[m] s_{i} & =0 \text { for } m \geqq 2 \text { and } j=1, \ldots, r . \quad \square
\end{aligned}
$$

From $L_{-1}|0\rangle=0$ and Theorem 5.1, we can easily find the proof of this.

5.3. Till now, we have regarded $z$ and $w$ as formal variables. But, in the following, we have to consider $z$ and $w$ as complex parameters. Let $V$ be a vector space and $\lambda$ in $\mathfrak{h}^{*}$. For $m=\left(m_{1}, \ldots, m_{N}\right) \in \mathbb{Z}^{N}$, let $A_{m}$ be a linear map from $V$ to $\mathscr{F}_{\lambda}$. 
Put $A(z):=\sum_{m \in \mathbb{Z}^{N}} z^{-m} A_{m}$, where we use the notations $z:=\left(z_{1}, \ldots, z_{N}\right)$ and $z^{-m}:=$ $z_{1}^{-m_{1}} \cdots z_{N}^{-m_{N}}$. We say that $A(z)$ converges at $z \in \mathbb{C}^{N}$, if the following infinite sum converges at $z$ for every $u \in \mathscr{F}_{\lambda}^{\dagger}$ and $v \in V$ :

$$
\langle u \mid A(z) v\rangle:=\sum_{m \in \mathbb{Z}^{N}} z^{-m}\left\langle u \mid A_{m} v\right\rangle .
$$

Then $A(z)$ is regarded as a linear functional on $\mathscr{F}_{\lambda}^{\dagger} \otimes V$. Of course, the operators introduced in the present paper converge in $\mathbb{C}^{\times}=\{z \in \mathbb{C} \mid z \neq 0\}$, for example,

$$
x_{\alpha}(z), \delta_{\alpha}(z), p_{i}(z), X(z), T(z), V(\lambda ; z), S_{i}(z), s_{i}(z) \text {, etc., }
$$

where $\alpha \in \Delta_{+}, X \in \mathfrak{g}, \lambda \in \mathfrak{h}^{*}$ and $i=1, \ldots, r$. However we should pay attention to the fact that $V(\lambda ; z)$ and $s_{i}(z)$ are multivalued in $z$. Put $w:=\left(w_{1}, \ldots, w_{L}\right)$ and $U^{L}:=\left\{w \in \mathbb{C}^{L} \mid w_{i} \neq w_{j}\right.$ if $\left.i \neq j\right\}$. For $i=1, \ldots, L$, let $A_{i}(z)$ be one of the operators in (5.24). Then the composition $A_{1}\left(w_{1}\right) \cdots A_{L}\left(w_{L}\right)$ converges in $\left\{\left|w_{1}\right|>\cdots>\left|w_{M}\right|>0\right\}$ and is analytically prolongable to $w$ in $U^{L}$. We denote the result of this prolongation by $\prod_{i=1}^{L} A_{i}\left(w_{i}\right)$. Furthermore, if $\left\{A_{i}(z)\right\}_{i=1}^{L}$ contains neither $V(\lambda ; z)$ nor $s_{i}(z)$, then $\prod_{i=1}^{L} A_{i}\left(w_{i}\right)$ is single-valued in $w$ and independent of the order of the composition. On the other hand, if $\left\{A_{i}(z)\right\}_{i=1}^{L}$ contains $V(\lambda ; z)$ or $s_{i}(z)$, then $\prod_{i=1}^{L} A_{i}\left(w_{i}\right)$ is, in general, multivalued in $w$ and is independent of the order of the composition if we ignore its phase factor.

5.4. Let us define conformal blocks, which is the most fundamental objects in conformal field theories.

Definition. Let $\lambda_{1}, \ldots, \lambda_{N}$ and $\lambda_{\infty}$ be in $\mathfrak{h}^{*}$ and $\Phi$ a linear map from $\mathscr{F}_{\lambda_{x}}^{\dagger} \otimes \bigotimes_{a=1}^{N} \mathscr{F}_{\lambda_{a}}$ to the space of multivalued regular functions on $U^{N}$. Then $\Phi$ is called a conformal block (of the WZW model on $\mathbb{P}^{1}$ ) for $\mathscr{F}_{\lambda_{\infty}}^{\dagger} \otimes \bigotimes_{a=1}^{N} \mathscr{F}_{\lambda_{a}}$, if it satisfies the following
conditions:

(B1) Let $A(\zeta)$ be one of the operators $T(\zeta)$ and $X(\zeta)$ for $X \in \mathfrak{g}$. For a fixed $z=$ $\left(z_{1}, \ldots, z_{N}\right) \in U^{N}$, let $f(\zeta)$ be a rational function regular in $\left\{\zeta \in \mathbb{C} \mid \zeta \neq z_{1}, \ldots, z_{N}\right\}$. For $u_{a} \in \mathscr{F}_{\lambda_{a}}, u_{\infty}^{\dagger} \in \mathscr{F}_{\lambda_{\infty}}^{\dagger}$ and $a=1, \ldots, N$, put

$$
\begin{aligned}
r_{a} & :=\operatorname{Res}_{\zeta=z_{a}}\left(f(\zeta) A\left(\zeta-z_{a}\right) d \zeta\right) u_{a}, \\
r_{\infty}^{\dagger} & :=u_{\infty}^{\dagger} \operatorname{Res}_{\zeta=\infty}(f(\zeta) A(\zeta) d \zeta), \\
v_{a} & :=u_{\infty}^{\dagger} \otimes u_{1} \otimes \cdots \otimes r_{a} \otimes \cdots \otimes u_{N}, \\
v_{\infty} & :=r_{\infty}^{\dagger} \otimes u_{1} \otimes \cdots \otimes u_{N},
\end{aligned}
$$

where $r_{a}$ in $v_{a}$ is placed at the $a^{\text {th }}$ component. Then we have

$$
\Phi\left(\sum_{a=1}^{N} v_{a}+v_{\infty} ; z\right)=0 \text {. }
$$


(B2) For $u_{a} \in \mathscr{F}_{\lambda_{a}}, u_{\infty}^{\dagger} \in \mathscr{F}_{\lambda_{x}}^{\dagger}$ and $a=1, \ldots, N$, we have

$$
\Phi\left(v_{a} ; z\right)=\frac{\partial}{\partial z_{a}} \Phi(u ; z)
$$

where we put $u:=u_{\infty}^{\dagger} \otimes u_{1} \otimes \cdots \otimes u_{N}$ and

$$
v_{a}:=u_{\infty}^{\dagger} \otimes u_{1} \otimes \cdots \otimes L_{-1} u_{a} \otimes \cdots \otimes u_{N} .
$$

Note. Physicists maybe prefer to use the following notation for conformal blocks:

$$
\left\langle u_{\infty}^{\dagger}(\infty) u_{1}\left(z_{1}\right) \cdots u_{N}\left(z_{N}\right)\right\rangle_{\Phi},=\Phi\left(u_{\infty}^{\dagger} \otimes u_{1} \otimes \cdots \otimes u_{N} ; z\right),
$$

where $u_{a} \in \mathscr{F}_{\lambda_{a}}$ and $u_{\lambda_{x}}^{\dagger} \in \mathscr{F}_{\lambda_{x}}^{\dagger}$.

Lemma 5.3. Let $\mu_{1}, \ldots, \mu_{L-1}$ and $\mu_{\infty}$ be in $\mathfrak{h}^{*}$. Let $\Psi$ be a conformal block for $\mathscr{F}_{\mu_{\infty}}^{\dagger} \otimes$ $\bigotimes_{a=1}^{L} \mathscr{F}_{v_{a}}$ and $u^{\prime}$ in $\mathscr{F}_{\mu_{x}}^{\dagger} \otimes \bigotimes_{a=1}^{L-1} \mathscr{F}_{\mu_{a}}$. Suppose that $\mu_{L}=-\alpha_{i}$ for some $i=1, \ldots$, r. For a fixed $w \in U^{L}$, let $f(\zeta)$ be a rational function on $\mathbb{C}$ regular at $\zeta=w_{L}$. Then we have the following:

$$
\begin{aligned}
\Psi\left(u^{\prime} \otimes r_{1} ; w\right) & =\frac{\partial}{\partial w_{L}}\left\{f\left(w_{L}\right) \Psi\left(u^{\prime} \otimes s_{i} ; w\right)\right\}, \\
\Psi\left(u^{\prime} \otimes r_{2}(X) ; w\right) & =0 \quad \text { for } \quad X \in \mathfrak{b}_{+}, \\
\Psi\left(u^{\prime} \otimes r_{2}\left(F_{j}\right) ; w\right) & =-\kappa \delta_{i, j} \frac{\partial}{\partial w_{L}}\left\{f\left(w_{L}\right) \Psi\left(u^{\prime} \otimes\left|-\alpha_{i}\right\rangle ; w\right)\right\} \quad \text { for } \quad j=1, \ldots, r,
\end{aligned}
$$

where we put

$$
\begin{aligned}
r_{1} & :=\operatorname{Res}_{\zeta=w_{L}}\left(f(\zeta) T\left(\zeta-w_{L}\right) d \zeta\right) s_{i}, \\
r_{2}(Y) & :=\operatorname{Res}_{\zeta=w_{L}}\left(f(\zeta) X\left(\zeta-w_{L}\right) d \zeta\right) s_{i} \quad \text { for } \quad Y \in \mathfrak{g} .
\end{aligned}
$$

This follows from Corollary 5.2 and the definition of conformal blocks.

For $\mu_{1}, \ldots, \mu_{L} \in \mathfrak{h}^{*}$, put $\vec{\mu}:=\left(\mu_{1}, \ldots, \mu_{L}\right)$ and $\mu_{\infty}:=\sum_{a=1}^{L} \mu_{a}$. The operator $V(\vec{\mu} ; w)$ defined by

$$
V(\vec{\mu} ; w):=: \prod_{a=1}^{L} e^{q\left(\mu_{a} ; w_{a}\right)} \cdot \prod_{1 \leqq a<b \leqq L}\left(w_{a}-w_{b}\right)^{\left(\mu_{a} \mid \mu_{b}\right) / \kappa},
$$

converges in $U^{L}$. The following formula is widely known:

$$
\prod_{a=1}^{L} V\left(\mu_{a} ; w_{a}\right)=V(\vec{\mu} ; w) \text { up to phase factor. }
$$

Using the method in [TK2], we can prove the following two lemmas.

Lemma 5.4. Under the above notation, there is a unique conformal block $\Psi$ of the WZW model for $\mathscr{F}_{\mu_{\infty}}^{\dagger} \otimes \bigotimes_{a=1}^{L} \mathscr{F}_{v_{a}}$ with the following properties: 
(1) Putting $v_{0}:=\left|\mu_{1}\right\rangle \otimes \cdots \otimes\left|\mu_{L}\right\rangle, u_{0}:=|0\rangle$ and $u_{\infty}^{\dagger}:=\left\langle\lambda_{\infty}\right|$, we have

$$
\Psi\left(u_{\infty}^{\dagger} \otimes v_{0} ; w\right)=\left\langle u_{\infty}^{\dagger} \mid V(\vec{\mu} ; w) u_{0}\right\rangle=\prod_{1 \leqq a<b \leqq L}\left(w_{a}-w_{b}\right)^{\left(\mu_{a} \mid \mu_{b}\right) / \kappa} .
$$

(2) The condition (B1) also holds, even if $A(\zeta)$ is equal to one of the operators $x_{\alpha}(\zeta)$, $\delta_{\alpha}(\zeta)$ and $p_{i}(\zeta)$ where $\alpha \in \Delta_{+}$and $i=1, \ldots, r$.

Lemma 5.5. Under the same situation as in Lemma 5.4, suppose that $L=N+M$ and $\mu_{N+b}=-\alpha_{\tau(b)}$ for some $\tau(b) \in \mathbb{N}$ and any $b=1, \ldots, M$. Let $I_{a}(\alpha)$ be in $\mathbb{N}$ for $a=1, \ldots, N$ and $\alpha \in \Delta_{+}$. Put $u_{0}:=|0\rangle$ and

$$
\begin{aligned}
u_{a} & :=\prod_{\alpha \in \Delta_{+}} x_{\alpha}[0]^{I_{a}(\alpha)}\left|\mu_{a}\right\rangle \text { for } a=1, \ldots, N, \\
v & :=u_{1} \otimes \cdots \otimes u_{N} \otimes S_{\tau(1)} \otimes \cdots \otimes s_{\tau(L)}, \\
\psi(w) & :=\prod_{a=1}^{N} \prod_{\alpha \in \Delta_{+}} x_{\alpha}\left(w_{a}\right)^{I_{a}(\alpha)} \prod_{b=1}^{M} S_{\tau(b)}\left(w_{N+b}\right) \prod_{a=1}^{L} V\left(\mu_{a} ; w_{a}\right) .
\end{aligned}
$$

Then, for $u_{\infty}^{\dagger} \in \mathscr{F}_{\mu_{\infty}}^{\dagger}$, the conformal block $\Psi$ in Lemma 5.4 satisfies the following:

$$
\Psi\left(u_{\infty}^{\dagger} \otimes v ; w\right)=\left\langle u_{\infty}^{\dagger} \mid \psi(w) u_{0}\right\rangle \text { up to phase factor }
$$

5.5. Let us construct integral representations of conformal blocks for $\mathscr{F}_{\lambda_{\infty}}^{\dagger} \otimes \bigotimes_{a=1}^{N} \mathscr{F}_{\lambda_{a}}$. For this purpose, we shall freely use the notation in 0.6 to 0.8 . For example, $\left(\lambda_{1}, \ldots, \lambda_{N}\right) \in\left(\mathfrak{h}^{*}\right)^{N}$ and $\lambda_{\infty}=\sum_{a=1}^{N} \lambda_{a}-\sum_{i=1}^{r} m_{i} \alpha_{i}$ for some $m_{i} \in \mathbb{N}$. Put $M:=\sum_{i=1}^{r} m_{i}$ and $L:=N+M$. Define $w=(z, t)$ by $(0.25)$ and $\vec{\mu}$ by $(0.26)$. Let $\Psi$ be the conformal block for $\mathscr{F}_{\mu_{\infty}}^{\dagger} \otimes \bigotimes_{a=1}^{L} \mathscr{F}_{v_{a}}$ in Lemma 5.3. Define the multivalued function $l(w)$ by (0.27). Then (5.32), (5.33) and Lemmas 5.4 (2) implies that, for each $v \in \mathscr{F}_{\mu_{\infty}}^{\dagger} \otimes \bigotimes_{a=1}^{L} \mathscr{F}_{{ }_{v}}$, the function $\Psi(v ; w)$ can be represented in the form $\Psi(v ; w)=l(w) f(w)$, where $f(w)$ is some rational function regular in $U^{L}$. Let $\{\Gamma(z)\}$ be a family of $M$-cycles with properties (0.28) and (0.29). Define the linear map $\Phi$ from $\mathscr{F}_{\lambda_{\infty}}^{+} \otimes \bigotimes_{a=1}^{N} \mathscr{F}_{\lambda_{a}}$ to the space of multivalued function on $U^{N}$ by

$$
\Phi(u ; z):=\int_{\Gamma(z)} \Psi(u \otimes s ; z, t) d t \quad \text { for } \quad u \in \mathscr{F}_{\lambda_{\infty}}^{\dagger} \otimes \bigotimes_{a=1}^{N} \mathscr{F}_{\lambda_{a}},
$$

where we put

$$
s:=s_{\tau(1)} \otimes \cdots \otimes s_{\tau(M)} \quad \text { (a tensor product of the screening vectors), }
$$

under the notation $(0.24)$.

Theorem 5.6. Under the notation, above $\Phi$ is a conformal block for $\mathscr{F}_{\lambda_{\infty}}^{\dagger} \otimes \bigotimes_{a=1}^{N} \mathscr{F}_{\lambda_{a}}$.

Proof. It is easy to see from (0.29) that $\Phi$ satisfies (B2). Let $A(\zeta)$ be one of the operators $T(\zeta)$ and $X(\zeta)$ for $X \in \mathfrak{g}$. For a fixed $z=\left(z_{1}, \ldots, z_{N}\right) \in U^{N}$, let $f(\zeta)$ be a 
rational function regular in $\left\{\zeta \neq z_{1}, \ldots, z_{N}\right\}$. Under the notation (5.25), define the vectors $u$ and $v$ in $\mathscr{F}_{\lambda_{\infty}}^{\dagger} \otimes \bigotimes_{a=1}^{N} \mathscr{F}_{\lambda_{a}}$ by

$$
\begin{aligned}
& u:=u_{\infty}^{\dagger} \otimes u_{1} \otimes \cdots \otimes u_{N}, \\
& v:=\sum_{a=1}^{N} v_{a}+v_{\infty} .
\end{aligned}
$$

In addition, we put, for $b=1, \ldots, N$,

$$
\begin{aligned}
& r_{b}\left(t_{b}\right):=\operatorname{Res}_{\zeta=t_{a}}\left(f(\zeta) A\left(\zeta-t_{a}\right) d \zeta\right) s_{\tau(b)}, \\
& e_{b}\left(t_{b}\right):=s_{\tau(1)} \otimes \cdots \otimes r_{b}\left(t_{b}\right) \otimes \cdots \otimes s_{\tau(M)},
\end{aligned}
$$

where $r_{b}\left(t_{b}\right)$ in $e_{b}\left(t_{b}\right)$ is placed at the $b^{\text {th }}$ component. Define the vector $e(t)$ in $\bigotimes_{b=1} \mathscr{F}_{-\alpha_{\tau}(b)}$ by

$$
e(t):=\sum_{b=1}^{M} e_{b}\left(t_{b}\right)
$$

Since $\Psi$ is a conformal block for $\mathscr{F}_{\mu_{\infty}}^{\dagger} \otimes \bigotimes_{a=1}^{L} \mathscr{F}_{v_{a}}$, we find that

$$
\Psi(v \otimes s+u \otimes e(t) ; z, t)=0 .
$$

On the other hand, Lemma 5.3 implies that

$$
\int_{\Gamma(z)} \Psi\left(u \otimes e_{b}\left(t_{b}\right) ; z, t\right) d t=0 \quad \text { for } \quad b=1, \ldots, M .
$$

Thus it is proved that $\Phi$ satisfies $(\mathrm{B} 1): \Phi(v ; z)=0$.

5.6. Now we shall show that the integral representation (5.39) of a conformal block implies that of a solution of the $\mathrm{KZ}$ equation (0.23). Owing to Proposition 4.4, we can identify $\mathscr{F}_{\lambda}[0]$ and $\mathscr{F}_{\lambda}^{\dagger}[0]$ with $M_{\lambda}^{*}$ and $M_{\lambda}^{\dagger}$, respectively. Thus the operator $\Omega_{a, b}$ defined by (0.22) acts on $\bigotimes_{a=1}^{N} \mathscr{F}_{\lambda_{a}}[0]$ and $\bigotimes_{a=1}^{N} \mathscr{F}_{\lambda_{a}}^{\dagger}[0]$.

Lemma $5.7[\mathrm{KZ}]$. Let $\lambda_{1}, \ldots, \lambda_{N}$ and $\lambda_{\infty}$ be in $\mathfrak{h}^{*}$ and $\Phi$ a conformal block for $\mathscr{F}_{\lambda_{\infty}}^{\dagger} \otimes \bigotimes_{a=1}^{N} \mathscr{F}_{\lambda_{a}}$. Then, for $u_{a} \in \mathscr{F}_{\lambda_{a}}[0]=M_{\lambda_{a}}^{*}$ and $u_{\infty}^{\dagger} \in \mathscr{F}_{\lambda_{\infty}}^{\dagger}[0]=M_{\lambda_{\infty}}^{\dagger}$, the conformal block $\Phi$ satisfies the following conditions:

(1) For $X \in \mathfrak{g}$, putting

$$
v:=u_{\infty}^{\dagger} X[0] \otimes u_{1} \otimes \cdots \otimes u_{N}-\sum_{a=1}^{N} u_{\infty}^{\dagger} \otimes u_{1} \otimes \cdots \otimes X[0] u_{a} \otimes \cdots \otimes u_{N} .
$$

we obtain $\Phi(v ; z)=0$.

(2) For $a=1, \ldots, N$, we have

$$
\frac{\partial}{\partial z_{a}} \Phi\left(u_{\infty}^{\dagger} \otimes u^{\prime} ; z\right)=\sum_{\substack{1 \leqq b \leqq N \\ b \neq a}} \frac{\Phi\left(u_{\infty}^{\dagger} \otimes \Omega_{a, b} u^{\prime} ; z\right)}{z_{a}-z_{b}},
$$

where we put $u^{\prime}:=u_{1} \otimes \cdots \otimes u_{N}$. 
Proof. Let $X$ be in $\mathfrak{g}$ and put $A(\zeta):=X(\zeta)$. Then applications of $(\mathrm{B} 1)$ to the cases $f(\zeta)=1$ and $f(\zeta)=\left(\zeta-z_{a}\right)^{-1}$ respectively imply (1) and the following:

$$
\begin{aligned}
& \Phi\left(u_{\infty}^{\dagger} \otimes u_{1} \otimes \cdots \otimes X[-1] u_{a} \otimes \cdots \otimes u_{N} ; z\right) \\
& \quad=\sum_{\substack{1 \leqq b \leqq N \\
b \neq a}} \frac{\Phi\left(u_{\infty}^{\dagger} \otimes u_{1} \cdots \otimes X[0] u_{b} \otimes \cdots \otimes u_{N} ; z\right)}{z_{a}-z_{b}} .
\end{aligned}
$$

By Lemma 4.5, we obtain

$$
L_{-1} u_{a}=\frac{1}{\kappa} \sum_{p=1}^{\operatorname{dim} g} J^{p}[-1] J^{p}[0] u_{a} .
$$

Thus, by (B2), we obtain

$$
\begin{aligned}
& \frac{\partial}{\partial z_{a}} \Phi\left(u_{\infty}^{\dagger} \otimes u^{\prime} ; z\right)=\frac{1}{\kappa} \sum_{p=1}^{\operatorname{dim} g} \Phi\left(u_{\infty}^{\dagger} \otimes u_{1} \otimes \cdots \otimes J^{p}[-1] J^{p}[0] u_{a} \otimes \cdots \otimes u_{N}\right) \\
& =\frac{1}{\kappa} \sum_{\substack{\leq b \leq \leqq N \\
b \neq a}} \sum_{p=1}^{\operatorname{dim} g} \frac{\Phi\left(u_{\infty}^{\dagger} \otimes \cdots \otimes J^{p}[0] u_{a} \otimes \cdots \otimes J^{p}[0] u_{b} \otimes \cdots \otimes u_{N}\right)}{z_{a}-z_{b}} .
\end{aligned}
$$

Rewriting this by $\Omega_{a, b}$, we find (2).

Recall that the weight subspace $M_{\vec{\lambda}, \lambda_{\infty}}^{\dagger}$ has been defined by (0.19). Lemma 5.5 implies the following.

Lemma 5.8. Under the same notation as in 5.5, we have

$$
\Psi\left(\left\langle\lambda_{\infty}\right| \otimes v \otimes s ; z, t\right)=\langle P(z, t) \mid v\rangle \quad \text { up to phase factor for } v \in M_{\vec{\lambda}, \lambda_{\infty}}^{*} .
$$

Theorem 5.9. Under the notation in 0.8 , if $F(z)$ is defined by $(0.32)$, then $F(z)$ is valued in $S_{\lambda_{\infty}}^{\dagger}\left(M_{\vec{\lambda}}^{\dagger}\right)$ and satisfies the $\mathrm{KZ}$ equation (0.23).

Proof. Let $\Phi$ denote the conformal block defined by (5.39). Then, by Lemma 5.8, we obtain

$$
\Phi\left(\left\langle\lambda_{\infty}\right| \otimes v ; z\right)=\langle F(z) \mid v\rangle \quad \text { for } \quad v \in M_{\bar{\lambda}, \lambda_{\infty}}^{*} .
$$

Therefore the theorem follows from Lemma 5.7.

Acknowledgements. The author sincerely thanks R. Hotta for continuous encouragement. After completing this paper, the author received the preprint [ATY] and wishes to thank Y. Yamada for sending it. In [ATY], it is pointed out that the Ward identities for $\left\{S_{i}(z)\right\}$ give a simple proof of the integral formulas in [SV].

\section{References}

[A1] Aomoto, K.: On the structure of integrals of power product of linear functions. Sci. Paper Coll. Gen. Ed. Univ. Tokyo 27, 49-61 (1977)

[A2] Aomoto, K.: Gauss-Manin connection of integrals of difference products. J. Math. Soc. Japan 39, 191-208 (1987)

[ATY] Awata, H., Tsuchiya, A., Yamada, Y.: Bosonization proof of the SchechtmanVarchenko Integral Formulas for WZNW correlation functions. preprint (1990)

[BF] Bernard, D., Felder, G.: Fock representations and BRST cohomology in SL(2) current algebra. preprint (1989) 
[BMP] Bouwknegt, P., McCarthy, J., Pilch, K.: Quantum group structure in the Fock space resolutions of $\hat{s}(n)$ representations. Commun. Math. Phys. 131, 125-155 (1990)

[BPZ] Belavin, A. A., Polyakov, A. M., Zamolodchikov, A. B.: Infinite conformal symmetry in two-dimensional quantum field theory. Nucl. Phys. B241, 333-380 (1984)

[CE] Cartan, H., Eilenberg, S.: Homological algebra. Princeton, New Jersey: Princeton University Press 1956

[CF] Christe, P., Flüme, R.: The four point correlations of primary operators of the $d=2$ conformal invariant $S U(2) \sigma$-model with Wess-Zumino term. Nucl. Phys. B282, 466-496 (1987)

[DF1] Dotsenko, V1. S., Fateev, V. A.: Conformal algebra and multipoint correlation functions in 2D statistical models. Nucl. Phys. B240 [FS12], 312-348 (1984)

[DF2] Dotsenko, V1. S., Fateev, V. A.: Four-point correlation functions and the operator algebra in 2D conformal invariant theories with central charge $c \leqq 1$. Nucl. Phys. B251 [FS13], 691-734 (1985)

[DJMM] Date, E., Jimbo, M., Matsuo, A., Miwa, T.: Hypergeometric type integrals and the $s l(2, \mathbb{C})$ Knizhnik-Zamolodchikov equation. preprint (1990)

[FeFr1] Feigin, B., Frenkel, E.: Representation of affine Kac-Moody algebras, bosonization and resolutions. In: Brink, L., Friedan, D., Polyakov, A. M. (eds.), Physics and Mathematics of Strings. Memorial volume for Vadim Knizhnik, pp. 271-316. Singapore, New Jersey, London, Hong Kong: World Scientific 1990

[FeFr2] Feigin, B., Frenkel, E.: Affine Kac-Moody algebras and semi-infinite flag manifolds. Commun. Math. Phys. 128, 161-189 (1990)

[FeFu1] Feigin, B. L., Fuchs, D. B.: Verma modules over the Virasoro algebra. In: Lecture Notes in Mathematics, Vol. 1060, pp. 230-245. Berlin, Heidelberg, New York: Springer 1984

[FeFu2] Feigin, B. L., Fuchs, D. B.: Representations of the Virasoro algebra. In: Leites, D. (ed.), Seminar on Supermanifolds 5. Report of Dept. Math. Stockholm University N 25 (1986). To be published in: Representation of Infinite-dimensional Lie Groups and Lie Algebras. New York: Gordon and Breach,

[Fel] Felder, G.: BRST approach to minimal models. Nucl. Phys. B317, 215-236 (1989)

[Fu] Fuks, D. B.: Cohomology of infinite-dimensional Lie algebras. New York: Plenum Press 1986

[GMMOS] Gerasimov, A., Marshakov, A., Morozov, A., Olshanelsky, M., Shalashvili, S.: WessZumino-Witten model as a theory of free fields. III. The case of arbitrary simple group. preprint (1989)

[Kac] Kac, V. G.: Infinite dimensional Lie algebras (Second Edition). Cambridge, London, New York, New Rochelle, Melbourne Sydney: Cambridge University Press 1985

[Ko] Kohno, T.: Homology of local system on the complement of hyperplanes. Proc. Jpn Acad. 62, Ser. A, 144-147 (1986)

[KZ] Knizhnik, V. G., Zamolodochikov, A. B.: Current algebra and Wess-Zumino models in two dimensions. Nucl. Phys. B247, 83-103 (1984)

[Mat] Matsuo, A.: An application of Aomoto-Gelfand hypergeometric functions to the $S U(n)$ Knizhnik-Zamolodchikov equation. Commun. Math. Phys. 134, 65-77 (1990)

[Mar] Marshakov, A. V.: The Dotsenko-Fateev representation for Wess-Zumino-Witten models. Phys. Lett. B224, 141-144 (1989)

[SV] Schechtman, V. V., Varchenko, A. N.: Integral representations of $n$-point conformal correlators in the WZW model. Preprint Max-Planck-Insitute für Mathematik, MPI/89-51, Bonn, August (1989)

[TK1] Tsuchiya, A., Kanie, Y.: Fock representation of the Virasoro algebra. Publ. RIMS, Kyoto Univ. 22, 259-327 (1986)

[TK2] Tsuchiya, A., Kanie, Y.: Vertex operators in conformal field theory on $\mathbb{P}^{1}$ and monodromy representations of Braid groups. Adv. Stud. Pure. Math. 16, 297-372 (1987)

[VGZ] Vasil'ev, V. A., Gel'fand, I. M., Zelevinskii, A. V.: General hypergeometric functions on complex Grassmannian. Funct. Anal. Appl. 21, 19-31 (1987)

[W] Wakimoto, N.: Fock representations of the affine Lie algebra $A_{1}^{(1)}$. Commun. Math. Phys. 104, 605-609 (1986) 